\title{
Chronic kidney disease alters Pin1 phosphorylation and parathyroid hormone mRNA binding proteins leading to secondary hyperparathyroidism
}

\author{
Alia Hasan, Yael E. Pollak, Rachel Levin, Justin Silver, Nir London, Morris Nechama*, \\ Iddo Z. Ben-Dov* and Tally Naveh-Many* \\ *, these authors contributed equally
}

\begin{abstract}
Parathyroid hormone (PTH) regulates calcium metabolism and bone strength. Chronic kidney disease (CKD) leads to secondary hyperparathyroidism (SHP) which increases morbidity and mortality. High PTH expression in SHP is due to increased PTH mRNA stability mediated by changes in PTH mRNA interaction with stabilizing AUF1 and destabilizing KSRP. Pin1 isomerizes target proteins, including mRNA binding proteins. In SHP, Pin1 isomerase activity is decreased and phosphorylated KSRP fails to bind PTH mRNA, resulting in high PTH mRNA stability and levels. The molecular mechanisms underlying Pin1 regulation and their effect to increase PTH expression are unknown. We show by mass-spectrometry (MS) the CKD induced changes in rat parathyroid proteome and phosphoproteome profiles. Parathyroid Pin1 Ser16 and Ser71 phosphorylation, that disrupts Pin1 activity, is enhanced in acute and chronic kidney failure rats. Accordingly, pharmacologic Pin1 inhibition increases PTH expression in parathyroid organ cultures and transfected cells, through the PTH mRNA protein binding cis element and KSRP phosphorylation. Therefore, CKD leads to parathyroid loss of Pin 1 activity by inducing Pin1 phosphorylation. This predisposes parathyroids to increase PTH production through modified PTH mRNA-KSRP interaction that is dependent on KSRP phosphorylation. CKD induced Pin1 and KSRP phosphorylation and the Pin1-KSRP-PTH mRNA axis thus drive secondary hyperparathyroidism.
\end{abstract}




\section{Introduction}

Parathyroid hormone (PTH) is central to calcium homeostasis and bone strength.

Changes in extracellular calcium are sensed by the parathyroid calcium sensing receptor (Casr), which in turn regulates PTH secretion and synthesis (1-4). Secondary hyperparathyroidism (SHP) is common in chronic kidney disease (CKD) and when poorly controlled, results in increased incidence of bone fractures, vascular calcification and mortality (5-7). SHP also occurs in acute kidney injury (AKI), because of abrupt deterioration in kidney function, and is frequently complicated by other mineral metabolism disorders (8-10). In experimental models of SHP due to dietary induced hypocalcemia or CKD, there are high PTH serum and mRNA levels, mainly due to posttranscriptional upregulation. The increase in PTH mRNA stability and steady-state levels correlates with changes in the binding of trans acting proteins, AUF1 (AU rich binding factor 1) and KSRP (K-homology splicing regulatory protein) to a defined evolutionary conserved adenine/uridine (AU) rich element (ARE) in the PTH mRNA 3'-untranslated region (3'-UTR) (11). The peptidyl prolyl cis/trans isomerase (PPIase) Pin1 coordinates these mRNA-protein interactions by regulating phosphorylation induced protein conformation (12).

AUF1 (encoded by HNRNPD) recognizes ARE sequences in mRNA 3'-UTRs inducing either target mRNA stability or decay (13-15). KSRP promotes rapid decay of ARE-containing RNAs. KSRP contains four contiguous K homology (KH) domains that recognize AREs and mediate RNA binding, mRNA decay, and interactions with the exosome and poly(A)-specific ribonuclease (PARN) (16). KSRP phosphorylation links KSRP-mediated mRNA degradation to extracellular signals (17). p38 MAPK-mediated KSRP Thr692 phosphorylation impairs KSRP-RNA interactions and increases abundance of a variety of target mRNAs (17-19). We have previously identified an additional human KSRP phosphorylation site, Ser181, which prevents KSRP mediated PTH mRNA degradation (12). Ser181 KSRP phosphorylation and Pin1 activity were also reported in the PTH-induced rapid decay of the type IIa sodium-phosphate cotransporter (Npt2) mRNA in renal opossum kidney cells (20).

Pin1 binds phosphorylated Ser/Thr residues followed by proline (pSer/Thr-Pro) in target proteins (21). This interaction catalyzes the cis-trans isomerization of the peptide bond, thereby changing the activity, stability and localization of target proteins participating in many cellular functions, including transcription and post-transcriptional regulation of gene expression (21-24). Pin1 interacts with several RNA binding proteins, 
among them KSRP, AUF1, HuR, Histone stem-loop-binding protein (SLBP) and cytoplasmic polyadenylation element binding protein (CPEB), that regulate mRNA decay, as well as other aspects of mRNA lifecycle (12, 25-28). These interactions depend on cell type and environmental stimuli. Pin1 interacts with KSRP in parathyroid glands and in human embryonic kidney (HEK) 293 cells (12). Pin1-mediated KSRP Ser181 dephosphorylation favors KSRP-PTH mRNA 3'-UTR binding and thus PTH mRNA decay. In SHP, parathyroid Pin1 isomerase activity is decreased and phosphorylated Ser181 KSRP fails to induce PTH mRNA decay, resulting in increased PTH mRNA and serum levels $(12,29,30)$.

Pin1 is composed of an N-terminal WW protein interaction domain and a Cterminal catalytic PPIase domain (21, 31-33). Pin1 binding and catalytic activity is tightly controlled by several Pin1 post-translational modifications. Protein kinase A (PKA) and S6 Kinase 2 (S6K2)-mediated Pin1 serine (Ser)16 phosphorylation abolishes the interaction of Pin1 with its substrates (34). Death-associated protein kinase 1 (DAPK1) phosphorylates Pin1 at Ser71 in the catalytic active site and inhibits its isomerase activity $(35,36)$. Therefore, Pin1 Ser16 or Ser71 phosphorylation is central to Pin1 inactivation. In addition, Pin1 cysteine (Cys)113 residue is essential for Pin1 catalytic activity and its oxidation inhibits Pin1 cellular function to promote tau and amyloid precursor protein turnover in neurons (37). Mutagenesis of Cys113 to alanine (Ala) results in loss of Pin1 protein activity (33). Screening an electrophilic fragment library targeting Cys113, identified sulfopin as a highly selective Pin1 inhibitor directed towards Pin1's active site. Sulfopin exhibits potent target engagement and phenocopies Pin1 genetic knockout in mice (38).

The molecular mechanisms that regulate Pin1 activity towards its target proteins are not fully understood. In particular, it is not clear what steers the decrease in Pin1 activity leading to high PTH levels in SHP $(12,39)$. We now show, using experimental models of chronic and acute kidney failure, parathyroid glands in culture, transfected cells and human parathyroid tissue that CKD alters overall parathyroid protein expression and phosphorylation. In particular, CKD leads to parathyroid specific Pin1 phosphorylation that is consistent with decreased Pin1 isomerase activity and the resulting parathyroid KSRP phosphorylation at Pin1 target motifs. Pharmacologic Pin1 inhibition, that mimics the decreased Pin1 activity in CKD, increases $P T H$ expression in parathyroid glands in culture and in transfected cells in a manner that is dependent on KSRP phosphorylation and the PTH mRNA cis acting element. Finally, end stage renal 
disease (ESRD) induces changes in human parathyroid phosphorylated Pin1 intracellular localization that could contribute to the increased PTH levels in these SHP patients. We suggest that CKD-induced Pin1 and hence KSRP phosphorylation and the Pin1-KSRPPTH mRNA axis are central to the increased PTH expression of SHP.

\section{Results}

\section{Chronic kidney disease induces changes in parathyroid proteome and phosphoproteome composition}

To characterize molecular changes in chronic kidney disease (CKD) induced secondary hyperparathyroidism (SHP), we performed, the first proteome and phosphoproteome analysis of the minute micro-dissected parathyroid tissue from CKD and control rats. Male rats were fed either a control or an adenine-rich high phosphorus diet for 2 weeks to induce CKD (12). At the end of the diet period, the two parathyroid glands of each rat were micro-dissected from surrounding thyroid tissue and pools of 2 rats were analyzed by mass spectrometry (MS) in triplicates. MS proteome analysis identified 4950 proteins groups, encoded by 3790 genes (several identifiers per gene often arising from splice isoforms). CKD parathyroid tissues could be distinguished from controls based on the overall proteome profiles (Fig 1A). Specifically, 173 and 110 proteins were up- and down-regulated, respectively, in extracts obtained from CKD induced SHP glands compared to control glands (Fig 1B and Supplemental Table 1). These changes in parathyroid gland protein expression highlight potentially modified pathways in CKD (Supplemental Fig 1), leading to SHP. As can be expected, hyperparathyroidism (hyperplastic state) was accompanied by changes in ribosome protein levels, protein translation, mRNA stability and secretion pathways (Fig 1C). Proteome analysis thus revealed moderate yet significant profile alteration in SHP and put forward pathways for future studies.

Phosphoproteome analysis of pools of parathyroid extracts from 6 control and 6 CKD rats recovered 5370 parathyroid phosphopeptides [87.3\% pSerine (Ser), 12.0\% pThreonine (Thr) and 0.7\% pTyrosine (Tyr)], encompassing 3884 phosphosites in 2222 phosphoproteins. Results of biological theme or function enrichment analysis of the identified phosphoproteins, with the detectable proteome as background using DAVID, are shown in Supplemental Fig 2A. Protein phosphorylation and kinase activity and structure terms dominate the enrichment lists, and transcription regulation is also prominent. This result is consistent with previous studies (40) demonstrating that 
regulatory proteins, such as transcription factors and kinases, are more often subjected to post-translational regulation via phosphorylation than are metabolic enzymes.

Enrichment of ErbB and mTOR signaling-related proteins is also apparent and warrants further studies in this model. The enrichment for mTOR signaling is consistent with our previous findings showing that mTOR activation is central to the development of SHP and parathyroid cell proliferation induced by either CKD or hypocalcemia in rats (41).

We then examined up- and downregulation of phosphosites and phosphoproteins. The detected phosphoproteome was subjected to phosphosite- and gene-centered enrichment analyses by means of a contemporary approach, 'PhosR' (42). To this end, we processed an additional pool of parathyroid glands from adenine-rich high phosphorus diet fed CKD rats and a pool of glands from control rats, and only phosphopeptides identified in both runs were included in the subsequent analysis ( $\mathrm{n}=4171$, Supplemental Table 2). Phosphosite dysregulation in adenine compared to control rats is shown in Fig 2A. The median $\log _{2}$ fold change of phosphosite abundance was 0.14 ( $10.5 \%$ increase), significantly higher than 0 (no change) according to a onesample Wilcoxon test $(\mathrm{p}<0.00001)$. Consistent with this shift, there were 460 significantly upregulated phosphosites (11.0\%) and only 348 significantly downregulated sites $(7.4 \%)$, indicating a possible global increase in protein phosphorylation. This may be expected in light of increased intracellular phosphate concentration in uremic SHP (43). However, we observed downregulation of the sodium-dependent phosphate transporter, Pit1, encoded by Slc20al ( $\log 2$ fold change $-1.17, \mathrm{p}<0.01)$, and respectively reduced Pit1;Ser269 and Pit1;Ser271 phosphorylation. In this regard, possible increases in Mapk1/3 (Erk1/1) activity, proposed to transduce the downstream signaling of high phosphate levels sensed by unknown mechanisms (that may include Pit1 and the calcium sensing receptor, Casr) (44) is suggested by enrichment of a potential MAPK phosphorylation motif $(45,46)<\mathrm{PLpSP}>$ among the recovered phosphosites in the adenine (CKD) pools (median $\log _{2}$ fold change $0.22, \sim 16.3 \%$ increase) (Supplemental Fig 2B). Thus, overall the phosphoproteome analysis suggests a globally increased phosphorylation state.

PhosR prioritizes potential kinases that could be responsible for the phosphorylation change of phosphosites based on kinase recognition motif and phosphoproteome dynamics (42). Kinase activity scores that sum changes in phosphorylation of targets, for which the targeting kinase is prioritized with a probability score, pointed to increased activity of specific kinases in adenine (CKD) (Supplemental Fig 2B). Among others, 
active (phosphorylated) human parathyroid Calcium/calmodulin-dependent protein kinase type II (Camk2a) has been shown to block PTH secretion ex-vivo and negatively correlate with serum calcium levels in patients with primary hyperparathyroidism, and thus may act to oppose hypersecretion (47), but studies in SHP are lacking. PKA (Prkaca) inactivates the isomerase Pin1 by phosphorylating Pin1 Ser16 in the WW domain, leading to dissociation of Pin1 from its substrates (34). Indeed, parathyroid Pin1 activity is decreased in SHP induced by either CKD or prolonged hypocalcemia (12). Numerous protein targets for Prkg1 (cGMP-dependent protein kinase 1) phosphorylation are implicated in modulating cellular calcium levels.

Gene-centered enrichment analysis of collapsed phosphosites showed multiple pathways significantly disturbed by uremic hyperparathyroidism (Supplemental Fig 2C). Of note, components of G-protein coupled receptor binding, signaling and GTPase cycles were enriched with hyper-phosphorylated proteins, while gene lists relating to cell cycle checkpoint and associated terms and events as well as negative regulation of FGFR signaling were enriched with under-phosphorylated proteins (i.e., enriched in the control glands).

Finally, applying the "Two Sample Logo" approach (48) we found global differences between adenine fed CKD rat parathyroid proteins with enriched and depleted phosphosites (Fig 2B). Proline (Pro) in the position after phosphorylated Ser, Thr or Tyr was markedly depleted in glands from adenine fed CKD rats (odds ratio 0.79, Bonferroni-corrected p-value <0.0001). Phosphorylated Ser/Thr-Pro motifs are potential targets for Pin1 that often leads to conformational changes that induce decreased phosphorylation of these sites in target proteins. Our current findings of reduced pSer/pThr followed by Pro imply fewer potential Pin1 targets in CKD rat parathyroid extracts, that could be due to changes in kinase activity, leaving Pin1 with less potential targets. The inset shows additional, less robust findings from this analysis (Fig 2B). In light of the latter result and our prior findings of decreased Pin1 activity in CKD parathyroids (12), we narrowed our search for proteins that were phosphorylated on Ser/Thr-Pro motifs as potential targets for Pin1 isomerase activity. We have previously shown that the PTH mRNA decay promoting protein KSRP is a target protein for Pin 1 . Pin1 over-expression induces KSRP dephosphorylation at Ser181 in transfected HEK293 cells, and decreases $P T H$ mRNA stability and levels (12). The decreased Pin1 activity in CKD parathyroids (12) could have an opposite effect, to increase KSRP phosphorylation. Indeed, CKD led to parathyroid KSRP Ser182 (Ser 181 in human) hyperphosphorylation 
in CKD rats (Fig 2C). In addition to Ser181, Pin1 phosphorylation at Thr101, was also increased ( $\mathrm{p}=0.016)$. KSRP pSer481, another yet uncharacterized potential Pin1 target, was decreased in parathyroids obtained from CKD rats $(\mathrm{p}=0.041$, Fig 2C). All these posttranslational modifications may be due to the decreased Pin1 activity in SHP parathyroids (12) and would alter KSRP-PTH mRNA interaction and contribute to the increased PTH mRNA levels in SHP. We therefore studied the changes that affect Pin1 isomerase activity in CKD-SHP and their effect on KSRP mediated PTH expression. Chronic and acute kidney failure induce parathyroid Pin1 specific phosphorylation that parallels decreased Pin1 activity in secondary hyperparathyroidism

Pin1 isomerase activity is regulated by phosphorylation at Ser16 and Ser71 that disrupts its interaction with target proteins and catalytic isomerase activity respectively (36). Because parathyroid Pin1 isomerase activity is decreased in CKD (12), we set out to determine if CKD affects parathyroid Pin1 phosphorylation. For that, we performed an in vitro kinase assay using recombinant GST-Pin1 and parathyroid extracts from control and CKD induced SHP rats. Rats were fed a control or an adenine-rich high phosphorus diet for 2 weeks that led to the expected increase in serum creatinine, phosphate and PTH levels (not shown) (12). Extracts from pools of micro-dissected parathyroid glands from control and CKD rats were incubated with recombinant GST-Pin1 or GST as control and $\left[\gamma-{ }^{32} \mathrm{P}\right] \mathrm{ATP}$, and analyzed after GST-pulldown by SDS-PAGE and autoradiography. SHP rat parathyroid extracts were more potent in inducing GST-Pin1 phosphorylation, compared to control extracts (Fig 3A\&B). Therefore, CKD leads to increased parathyroid phosphorylation activity towards Pin1.

We then examined parathyroid specific Pin1 Ser16 or 71 phosphorylation (Fig 3C), that disrupts Pin1 isomerase activity (36). We could not detect Pin1 pSer16 and pSer 71 containing peptides in our MS analysis, possibly due to limitations of the trypsin protein digestion method used (49). Immunofluorescence (IF) analysis of thyroparathyroid sections from CKD and control rats with specific targeted pSer16 and pSer 71 antibodies (35) showed increased phosphorylation of both Pin1 Ser 16 and 71 in CKD SHP parathyroid glands, compared to glands from control animals (Fig 3D-G). There was no change in total Pin1 protein levels, as expected (Supplemental Fig 3) (12).

To study Pin1 phosphorylation in an additional model of SHP, we induced acute kidney injury (AKI) by a single injection of high dose folic acid in mice $(9,10)$. Folic acid led to the expected increase in serum urea and PTH levels at 24 and $48 \mathrm{~h}$ (Supplemental Fig 4) (50). IF analysis showed that AKI mice had increased parathyroid 
Pin1 pSer16 and pSer71 phosphorylation compared to vehicle-injected mice (Fig 3H-K). As in CKD rat parathyroids, there was no change in total Pin1 levels (not shown). Therefore, SHP is characterized by increased parathyroid phosphorylation activity towards Pin1. Specifically, Pin1 Ser 16 and 71 phosphorylation is increased in two experimental models of chronic (CKD) and acute (AKI) SHP. The increased Ser 16 and 71 phosphorylation correlates with the decreased Pin1 activity and high PTH expression in SHP (12).

\section{PKA activation induces Pin1 Ser 16 phosphorylation and mediates the increase in PTH expression in parathyroid organ cultures and in transfected cells}

PKA, which is more active in uremic parathyroid glands (Supplemental Fig 2B), phosphorylates Pin1 Ser16 in the WW domain, leading to Pin1 dissociation from its substrate (34) (Fig 3C). To study the direct effect of PKA activation on PTH secretion, we used mouse thyro-parathyroids in organ culture, as there is no functional parathyroid cell line $(51,52)$. The specific PKA activator forskolin increased PTH accumulated in the culture media at 1 and 3 hours post incubation, compared to vehicle treated cells (Fig 4A). Therefore, PKA activation increases PTH secretion from parathyroid glands in culture.

We then studied the effect of PKA inhibition on PTH secretion. The specific PKA inhibitor, H89 (N-[2-p-bromocinnamylamino-ethyl]-5-isoquinolinesulphonamide) was added to rat micro-dissected parathyroid glands in culture. H89 led to a decrease in secreted PTH compared vehicle (DMSO) incubated glands (Fig 4B). We also added H89 to mouse thyro-parathyroids from control and adenine-rich high phosphorus induced CKD mice (Fig 4C). Of interest, thyro-parathyroids from CKD mice cultured in control medium for 7 and $24 \mathrm{~h}$, secreted more PTH compared to glands from mice with normal renal function, indicating that stimulation of the parathyroid by CKD in vivo is preserved in vitro (Fig 4C)(51). Importantly, H89 decreased PTH secretion from parathyroids in culture of both control and CKD mice (Fig 4C). The effect of PKA inhibition to decrease PTH secretion was also evident in folic acid induced AKI mouse thyro-parathyroid glands in culture (Fig 4D), similar to its effect on parathyroid glands form normal renal function and CKD mice and normal rats (Fig 4B-C). Therefore, PKA activation is central to the increase in PTH secretion in parathyroid glands from rats and mice with normal renal function and from mice with SHP induced by either CKD or AKI.

To further study the effect of PKA activation on $P T H$ expression, we used HEK293 cells transiently transfected with an expression plasmid carrying the human 
PTH gene $(12,53)$. Forskolin led the expected increase in Pin1 Ser16 phosphorylation in HEK293 cells (34) (Supplemental Fig 5). Importantly, forskolin increased PTH mRNA levels at both 3 and $24 \mathrm{~h}$ (Fig 4E), similar to its effect to increase PTH secretion from parathyroid glands in culture (Fig 4A). Altogether, these studies show that PKA activation that increases Pin1 Ser16 phosphorylation mediates the increased PTH expression in parathyroid glands in culture and in transfected HEK293 cells. These findings are consistent with increased parathyroid Pin1 phosphorylation in vivo (Fig 3) and the decreased Pin1 activity (12) in CKD SHP.

\section{Targeting the Pin1 catalytic domain by the pharmacological inhibitor Sulfopin increases $P T H$ expression in parathyroid organ cultures}

To further understand the role of Pin1 in mediating PTH expression, we studied the effect of Sulfopin, a highly selective nanomolar-range Pin1 inhibitor that targets Cys 113 in the catalytic domain (Fig 3C) $(33,38)$, in parathyroid organ cultures. For that we generated mice that express YFP specifically in their parathyroids (PT-YFP) (Fig 5A). After fluorescence-guided microsurgery, mouse parathyroid glands without the adjacent thyroid tissue were incubated in culture medium containing Sulfopin or vehicle (DMSO). Pin1 inhibition by Sulfopin led to an increase in both PTH secretion and PTH mRNA levels, compared to vehicle treated glands (Fig 5B-C). Altogether, our data show that Pin1 pharmacologic inhibition at both the binding and catalytic domains mimic the decreased Pin1 isomerase activity in SHP leading to high PTH levels.

Pin1 inhibition at either the WW or the catalytic domain increases PTH expression through the PTH mRNA 3'-UTR 63 nt protein binding element

A defined 63 nt ARE in the PTH mRNA 3'-UTR is both necessary and sufficient for protein-mRNA interaction and the regulation of PTH mRNA stability in SHP parathyroids and in vitro $(11,54)$. To determine if the effect of Pin1 inhibition to increase PTH expression is mediated by the PTH mRNA ARE, we transfected HEK293 cells with a wild type growth hormone (GH) expression plasmid or a $G H$ reporter plasmid containing the PTH mRNA 3'-UTR 63 nt cis-acting element (GH63) (Fig 6A) $(11,12,54)$. Forskolin increased $G H 63$ mRNA levels, similar to its effect to increase PTH mRNA, but had no effect on wild type GH that did not contain the PTH mRNA element (Fig 6B). Similarly, Pin1 inhibition by Sulfopin increased GH63 mRNA levels, but not wild type $G H$ mRNA levels (Fig 6C). Therefore, Pin1 inhibition induced by both PKA activation and Sulfopin increases $P T H$ expression through the PTH mRNA 3'-UTR $63 \mathrm{nt}$ protein-binding element. 


\section{Pin1 inhibition by both forskolin and Sulfopin alters over-all protein-PTH mRNA 3'-UTR interaction}

To characterize the PTH mRNA 3'-UTR binding proteins and in particular the effect of Pin1 inhibition on protein-RNA binding, we performed liquid chromatography-mass spectrometry (LC-MS) analysis of PTH mRNA 3'-UTR interacting proteins from HEK293 cells incubated with forskolin, Sulfopin or vehicle. Proteins were pulled-down using biotinylated in vitro-transcribed human PTH mRNA 3'-UTR immobilized onto streptavidin beads. The transcript-bound proteins were eluted and analyzed by LCMS/MS. Across all extracts, MS identified 610 proteins (post filtering of common contaminants), of which 82 proteins (13.4\%) were detected at levels significantly above the background recovered from uncoated streptavidin beads (Supplemental Fig 6A). The majority of these (53\%) were RNA binding proteins and many others were involved in mRNA regulation and processing (Fig 7A, and Supplemental Fig 6B). The top enriched RNA binding proteins were PRKRA (an RNA-dependent protein kinase activator) and IGF2BP1 (a well-studied mRNA-BP, which inhibits both mRNA translation and degradation, depending on its binding target). Additional highly enriched proteins were KSRP and AUF1 (HNRNPD), the two PTH mRNA-interacting proteins central for increased PTH expression in SHP $(11,14)$, confirming the validity of the assay. Of interest, Pin1 was also pulled-down specifically by the PTH mRNA 3'-UTR (Supplemental Fig 6A). Enrichment of several pulled-down proteins was boosted in extracts recovered from cells incubated with either of the Pin1 inhibitors, and the effect of inhibitors was largely similar (Fig 7B and Supplemental Fig 6C-D). The most significantly amplified proteins were APEH, NEU2, GLOD4, NAP114, ILF2/3, PRKRA, IGF2BP1/3 and STAU2. In the list of pulled-down proteins ( $\mathrm{n}=82$ significantly above background +73 borderline, see Supplemental Fig 6A), those proteins further intensified in the presence of Pin1 inhibitors $(n=31)$ were enriched with the following annotations: double-stranded RNA binding, protein binding, poly(A) RNA binding and intracellular ribonucleoprotein complex. Thus, Pin1 inhibition results in altered protein-PTH mRNA interactions. This is particularly interesting since Pin1 inhibition by forskolin and Sulfopin mimics the decreased Pin1 isomerase activity in parathyroids in SHP mice and rats (12).

\section{PKA activated Pin1 inhibition decreases Pin1-KSRP interaction}

To understand the effect of Pin1 inhibition on the $P T H$ mRNA decay promoting protein KSRP, we first studied Pin1-KSRP protein-protein interaction after Pin1 inhibition, by 
co-IP analyses. Cells were transiently transfected with FLAG-KSRP expression plasmid and then incubated with forskolin, Sulfopin or vehicle. At 24 h extracts were prepared and immunoprecipitated with Pin1 antibody. Western blots showed that KSRP was pulled-down by Pin1, as expected (Supplemental Fig 7) (12). Forskolin decreased Pin1FLAG-KSRP interaction, consistent with forskolin induced Pin1 Ser16 phosphorylation that targets the Pin1 protein-binding domain. Sulfopin acting on the catalytic domain of Pin1, had no effect on Pin1-KSRP interaction (Supplemental Fig 7). Therefore, PKA activation leads to dissociation of Pin1 from KSRP in HEK293 cells.

\section{Pin1 induced changes in KSRP phosphorylation alter PTH expression}

Phosphoproteome analysis showed that KSRP is hyper-phosphorylated at Ser181, and Thr101 in parathyroids from CKD rats (Fig 2C). We have previously shown that Pin1 over-expression leads to KSRP Ser181 dephosphorylation that promotes PTH mRNA decay (12). KSRP Thr692 phosphorylation impairs KSRP's decay-promoting activity on a variety of target mRNAs (55). Ser181, Thr101 and Thr692 all precede Pro as expected from target sites for Pin1 isomerase activity (Fig 8A). To characterize the impact of these KSRP pSer/Thr-Pro phosphorylation sites on PTH mRNA levels, HEK293 cells were cotransfected with expression plasmids for PTH and wt KHSRP or KHSRP phospho-null mutants. Co-transfection of PTH and wt KSRP expression plasmids led to the expected decrease in PTH mRNA (Fig 8B), consistent with the PTH mRNA decay promoting activity of KSRP (12). Interestingly, Ser181Ala, Thr100Ala and Thr692Ala single phosphorylation null mutants had a similar effect as wt KSRP to decrease $P T H$ mRNA, and forskolin only partially reversed this effect (Fig 8B\&C). In contrast to the KSRP single phosphorylation null mutants, the ability of wt KSRP to decrease PTH mRNA was magnified by overexpression of the KSRP Ser181Ala;Thr100Ala double mutant and more so by the Ser181Ala;Thr100Ala;Thr692Ala triple mutant (Fig 8D). Therefore, KSRP dephosphorylation at more than one Ser/Thr-Pro motif is required to maximize the KSRP mediated decrease in PTH expression. Forskolin activated PKA induced Pin1 phosphorylation, which would leave KSRP in its phosphorylated form (12), increased PTH mRNA levels when wt KSRP was over-expressed (Fig 8D). However, forskolin did not restore $P T H$ expression when the double or triple KSRP phosphorylation null mutants were over-expressed (Fig 8D). Therefore, the increase in $P T H$ expression by Pin1 inhibition is dependent on KSRP phosphorylation.

We next asked whether Pin1 inhibition by sulfopin and KSRP phosphorylation act via the GH-PTH63 (GH63) mRNA element. We therefore co-transfected cells with 
expression plasmids for GH63 and either KSRP or the KSRP

Ser181Ala;Thr100Ala;Thr692Ala triple phosphorylation null mutant. Sulfopin increased GH63 mRNA levels in wt KSRP overexpressing cells (Supplemental Fig 8), similar to its effect without KSRP over-expression (Fig 6C). Importantly, Pin1 inhibition failed to increase GH63 mRNA levels in cells over expressing KSRP triple phosphorylation null mutant (Supplemental Fig 8). Therefore, decreased Pin1 activity increases PTH expression in a manner that is dependent on KSRP phosphorylation at least in 2 phosphorylation sites. The Pin1-KSRP axis and the PTH mRNA 3'-UTR cis element are central to this increase in PTH expression. We suggest that in vivo, loss of Pin1 activity in SHP parathyroids increases PTH expression through modified PTH mRNA-KSRP interaction that is dependent on KSRP phosphorylation.

\section{Pin1 phosphorylation in human parathyroid sections from controls and patients} with advanced CKD

To determine the physiologic relevance of Pin1 mediated PTH regulation in patients with advanced CKD, we stained archival parathyroid sections, from end-stage renal disease (ESRD) patients and control parathyroids removed at total thyroidectomy. Of interest, IF staining showed that CKD parathyroids undergo morphologic changes where the nuclei of the parathyroid producing cells become eccentric, forming nodule-like structures (Fig 9). The significance of these structures remains to be determined. IF staining for Pin1 pSer16 and pSer71 showed their expression in the cytoplasm and nucleus in control parathyroids. In parathyroids from ESRD patients staining for both pSer16 and pSer 71was more intense in the nuclei, suggesting that there was a shift of pSer16 and pSer71 to the nucleus in CKD (Fig 9). This reproducible change in pSer16 and pSer71 intracellular localization was evident throughout the parathyroid sections. However, because of the heterogeneity of the human glands, it is difficult to determine if the intensity of phosphorylated Pin1 is indeed altered in human CKD parathyroids, as was evident in experimental models (Fig 3). We could not detect a similar shift to the nucleus in pSer16 and pSer71 Pin1 in rat and mouse CKD parathyroids (Fig 3). We suggest that CKD alters Pin1 pSer16 and pSer71 in parathyroids from ESRD patients and experimental models and this would lead to decreased Pin1 activity and altered protein$P T H$ mRNA interactions resulting in the high PTH gene expression of CKD induced SHP. 


\section{Discussion}

Secondary hyperparathyroidism (SHP) is a frequent complication and a key player in the devastating clinical consequences of CKD. Here we aim to elucidate the signaling pathways and underlying molecular mechanisms involved in the stimulated PTH synthesis and secretion in SHP. Mass spectrometry analysis identified changes in parathyroid proteome and phosphoproteome profiles induced by experimental CKD. To the best of our knowledge, this is the first attempt to characterize the global changes in protein expression and phosphorylation induced by CKD in the minute rodent parathyroid glands. Our data highlight new potential pathways that may be involved in the CKD induced changes in parathyroid function resulting in SHP.

Phosphoproteome analysis identified up- and down-regulated phosphosites, with more phosphosites upregulated than downregulated, suggesting an increase in global protein phosphorylation. Increased intracellular phosphate concentrations were reported in uremic SHP (43). Among others, we show enrichment of mTOR signalingrelated proteins. Indeed, mTOR activation is central to the development of SHP and parathyroid cell proliferation induced by either CKD or hypocalcemia in rats, confirming the validity of the assay (41). Kinase activity scores that sum changes in phosphorylation of target proteins, pointed to increased activity of several kinases in CKD parathyroids. Among them, PKA increased activity was demonstrated. PKA inactivates Pin1 isomerase activity by targeting Pin1 Ser16 phosphorylation in the WW domain, leading to dissociation of Pin1 from its substrates (34). In the context of PTH regulating pathways, PKA activation could mediate the decreased parathyroid Pin1 activity that occurs in SHP induced by either CKD or prolonged hypocalcemia (12). We then looked at global differences between parathyroid proteins from adenine fed CKD rat, with enriched and depleted phosphosites (Fig 2A). The change in potential phosphorylated Ser/Thr-Pro Pin1 target sites in CKD rat parathyroid extracts highlights a role for Pin 1 in SHP.

Among the proteins identified by the phosphoproteome, KSRP was phosphorylated in CKD parathyroids at residues that may be potential targets for Pin1 isomerize activity. We have previously shown that KSRP is a target protein for Pin1. Pin1 overexpression in transfected cells decreases KSRP phosphorylation at Ser181 which resulted in decreased $P T H$ mRNA stability and levels. The decreased Pin1 activity in CKD parathyroids (12) could increase KSRP phosphorylation. Indeed, CKD led to parathyroid KSRP Ser182 (Ser 181 in human KSRP) phosphorylation in CKD rats by 
phosphoproteome analysis. In addition to Ser181, Pin1 phosphorylation at Thr101, was also increased in CKD parathyroids. KSRP Ser481 phosphorylation, another potential Pin1 target, was decreased. These post-translational modifications may be due to the decreased Pin1 activity in SHP parathyroids and could alter KSRP-PTH mRNA interaction and contribute to the increased PTH mRNA levels in SHP. Of interest, Ser181 KSRP and Pin1 phosphorylation was also identified in PTH-stimulated opossum kidney (OK) proximal tubule cell culture model, by MS phosphoproteome characterization (20). It is intriguing that KSRP and Pin1 are involved in both the kidney and parathyroid, two organs central to mineral metabolism.

Recently, mRNA profiles of porcine parathyroid glands were performed in a long-term dietary phosphorus intervention, by keeping pig offspring on distinct mineral phosphorus levels throughout fetal and postnatal life. RNA sequencing data and resulting molecular pathways of parathyroid glands showed that PTH abundance is controlled via Pin1, CaSR, MAfB, PLC and PKA signaling to regulate $P T H$ expression, stability, and secretion. Parathyroid glands revealed lowered Pin1 mRNA abundance in animals fed a low phosphorus diet with no change in the expression of KSRP by post-weaning diets (56).

Based on the changes in KSRP phosphorylation by the phosphoproteome analysis and previous studies on KSRP and Pin1 in the parathyroid, we chose to study the parathyroid KSRP-Pin1 axis and its effects on PTH expression in CKD SHP. We show that both CKD and AKI lead to increased parathyroid Pin1 phosphorylation at Ser16 and 71, consistent with the decreased Pin1 isomerase activity $(35,36)$ and similar to the decreased Pin1 activity in parathyroids of rats with hypocalcemia or CKD induced SHP (12). We then studied the mechanisms of decreased Pin 1 activity in both parathyroid glands in culture and transfected cells, as there is no functional parathyroid cell line. Pharmacologic inhibition of Pin1 by PKA activation, which leads to Ser16 Pin1 phosphorylation at the protein binding WW domain, increased PTH expression in both parathyroid organ cultures and transfected cells. PKA inhibition had the opposite effect. Inhibition of Pin1 by targeting C113 in the catalytic domain with sulfopin had similar effects to increase $P T H$ expression. Therefore, pharmacologic inhibition, at both the WW and the catalytic domain, that mimics the decreased Pin1 activity in vivo in the CKD SHP parathyroids, leads to increased $P T H$ expression also when added directly in vitro. This effect was dependent upon the PTH mRNA 3'-UTR cis acting element. 
Forskolin has been shown to increase cellular cyclic adenosine monophosphate content and parathyroid hormone release in dispersed primary bovine parathyroid cells (57). Cholera toxin which activates adenylate cyclase and indirectly stimulates PKA, increased PTH mRNA and PTH secretion in dispersed bovine parathyroid cells (58). The mechanism of increased PTH expression by PKA activation was not studied at the time, but may be due to the activated PKA induced Pin1 Ser16 phosphorylation and decreased Pin1 activity, suggested by our current findings.

To understand the effect of Pin1 inhibition on PTH expression and mRNA stability, we identified by Mass spectrometry the PTH mRNA 3'-UTR interacting proteins and the effect of Pin1 inhibition on mRNA binding. We identified many proteins that are involved in mRNA fate, among them KSRP, AUF1 and Pin1.

KSRP over expression that induces PTH mRNA decay and decreased PTH expression in transfected cells (12) was more potent when KSRP phosphorylation null mutations at Pin1 potential target site were introduced. Moreover, the effect of Pin1 pharmacologic inhibition to increase $P T H$ expression was dependent upon KSRP phosphorylation at Ser181 and Thr100 and more so when an additional phosphorylation site Thr692 is present. p38 MAPK-mediated KSRP phosphorylation at Thr692 Pin1 potential site (pThr692-Pro693) impairs KSRP-RNA interactions and increases target mRNA abundance in a variety of target mRNA, demonstrating that KSRP phosphorylation is crucial for its mRNA decay promoting capabilities (26). These findings are consistent with the decreased Pin1 activity in vivo in SHP that correlates with increased PTH expression (12) and the increased KSRP phosphorylation at Ser181 and Thr100 shown in the phosphoproteome analysis (Fig 2C).

Finally, we show the pathophysiological relevance of our findings to CKD SHP, by changes in Pin1 pSer16 and pSer71 intracellular localization and possibly protein levels in parathyroid sections from ESRD patients. The changes in Pin1 phosphorylation induced by renal failure in human SHP parathyroids suggest that Pin1, together with KSRP phosphorylation, lead to increased PTH mRNA stability and serum levels in these patients, similar to the mechanisms in experimental models.

In summary, our findings show that CKD induces global changes in expression and phosphorylation of parathyroid proteins. Specifically, Pin1 is phosphorylated at residues that inhibit its isomerase activity, explaining the decreased Pin1 activity we have previously shown in SHP parathyroids (12). The decreased Pin1 activity and the resulting KSRP phosphorylation at Pin1 target sites would alter protein-PTH-mRNA 
interactions that ultimately increase PTH expression. These effects can be mimicked in vitro in parathyroid glands in culture and in transfected cells, by pharmacological inhibition of Pin1. We propose that Pin1 phosphorylation induced decrease in Pin1 activity increases PTH expression in SHP in a manner that is dependent on KSRP phosphorylation and the PTH mRNA cis acting element (Fig 10). Loss of Pin1 activity in SHP parathyroids triggers the parathyroids to increase PTH expression through modified PTH mRNA-KSRP interaction that is dependent on KSRP phosphorylation.

\section{Materials and Methods}

Animals, housing and diets

Chronic kidney disease (CKD) was induced in male Sprague-Dawley rats (150-170 g) by an adenine-rich $(0.75 \%)$ high-phosphorus (1.5\%) diet (ENVIGO, Harlan Laboratories, Madison, WI) given for $14 \mathrm{~d}$ (12). Controls received regular chow containing $0.7 \%$ phosphorus. CKD in mice was induced in male C57BL/6 mice at 11-12 wk of age by a moderate (0.3\%) adenine-rich high-phosphorus (1.2\%) diet (ENVIGO) given for $14 \mathrm{~d}(52,59)$. Acute kidney injury (AKI) was induced in male C57BL/6 mice at $11-12 \mathrm{wk}$ of age by a single injection of folic acid $(240 \mathrm{mg} / \mathrm{kg}$ in vehicle, $0.15 \mathrm{~mol} / \mathrm{l}$ NaHCO3, pH 7.4, Sigma-Aldrich, St. Louis, MO) (9, 10). Control mice were injected with vehicle. Mice were analyzed at 24 and $48 \mathrm{~h}$. In some experiments, PT-EYFP (parathyroid specific enhanced Yellow Fluorescent Protein) mice were used to allow identification and micro-dissection of the mouse parathyroid glands for organ culture experiments. We generated the PT-EYFP mice by Cre-Lox recombination, mating PTCre mice where the Cre recombinase is driven by the human PTH promoter [FVBTg(PT-Cre); Jackson Laboratory, Bar Harbor, ME] (2) with R26-stop-EYFP/R26R$E Y F P$, mice, where a loxP-flanked STOP sequence followed by the EYFP was inserted into the Gt(ROSA)26Sor locus (60). Total DNA was extracted from tail samples of offspring and genotyping was performed by PCR. The primers used were, for Cre: Fw: 5'-TGCCACGACCAAGTGACAGC-3'and Rev: 5'CCAGGTTACGGATATAGTTCATG-3' (52) and for EYFP: ROSA 26R 5'-AAAGTC GCTCTGAGTTGTTAT-3'; BTG 60: 5'-GAAAGACCGCGAAGAGTT TG-3' and BTG 62: 5'-TAAGCCTGCCCAGAAGACTC-3'. All animals had free access to food and drinking water. Experiments were approved by the Institutional Animal Care and Use Committee of the Hebrew University-Hadassah medical school (authorization number MD-18-15408, 18-15610). 


\section{Parathyroid glands in organ culture}

The 2 glands from micro-dissected mouse thyro-parathyroid tissue, parathyroid glands from PT-EYFP mice or rat parathyroids $(\mathrm{n}=6)$ were maintained in $2 \mathrm{ml}$ Eppendorf tubes with needle-punctured caps for aeration, containing $1 \mathrm{ml}$ DMEM (Gibco Life Technologies, Carlsbad, CA) supplemented with 10\% fetal bovine serum, L-glutamine, and penicillin-streptomycin (Gibco Life Technologies). Forskolin at a concentration of $100 \mu \mathrm{M}, \mathrm{H} 89$ (N-[2-p-bromocinnamylamino-ethyl]-5-isoquinolinesulphonamide) at 150 $\mu \mathrm{M}$, Sulfopin at $50 \mu \mathrm{M}$ or vehicle (DMSO) were added to the medium. The tubes were placed in a $\mathrm{CO}_{2}$ incubator with constant rocking $(52,61)$. Medium $(100 \mu 1)$ was collected at the time points indicated and analyzed for PTH. In some experiments, thyroidparathyroid glands were removed from adenine high-phosphorus diet induced CKD, $\mathrm{AKI}$ and control mice.

\section{$\underline{\text { Serum biochemistry and PTH levels }}$}

Serum was analyzed for calcium and blood urea nitrogen (BUN) using QuantiChrom kits (BioAssay Systems, Hayward, CA). Serum phosphate was analyzed using a Stanbio Phosphorus Liqui-UV kit (Stanbio Laboratories, Boerne, TX). Serum PTH or PTH secreted to growth medium in organ cultures was measured using rat or mouse 1-84 Intact PTH ELISA kit (Quidel, Athens, Ohio).

\section{$\underline{\text { RNA-protein extraction }}$}

Thyro-parathyroid glands in organ culture were removed from the medium at the end of the incubation period and homogenized using a bead-beater. RNA was extracted from pools of glands form 6 mice using TRIzol Reagent (Invitrogen, Carlsbad, CA). RNA from HEK293 cells was also extracted with TRIzol Reagent (12).

Protein extracts for immuno-precipitation (IP) in cultured HEK293 cells were prepared using RIPA buffer containing $150 \mathrm{mM} \mathrm{NaCl}, 1 \% \mathrm{NP} 40,0.5 \%$ sodium deoxycholate, $0.1 \%$ SDS and protease inhibitors cocktail (Roche, Mannheim, Germany). Antibodies used were Pin1 (Abcam, Cambridge MA) and FLAG, (Sigma-Aldrich). Mass Spectrometry (MS) for proteomics and phosphoproteomics was performed at the Technion Israel Institute of Technology, Haifa, Israel Pooled parathyroid tissue samples from 2 rats in every group in triplicate were homogenized in lysis buffer and sonicated. The proteins were digested in $1 \mathrm{M}$ urea with modified trypsin (Promega, Madison, WI) at a 1:50 enzyme-to-substrate ratio, overnight at $37^{\circ} \mathrm{C}$. The tryptic peptides were desalted and re-suspended in $40 \%$ acetonitrile $(\mathrm{ACN}), 6 \%$ TFA (Trifluoroacetic acid), and enriched for phosphopeptides on titanium 
dioxide beads. Bound peptides were eluted with $20 \%$ ACN with 325 mM Ammonium Hydroxide followed by $80 \%$ ACN with $325 \mathrm{mM}$ Ammonium Hydroxide. The resulting peptides were desalted and analyzed by LC-MS/MS on a Q- Exactive plus mass spectrometer (Thermo-Fisher scientific, Pittsburgh, PA) fitted with a capillary HPLC (easy nLC 1000, Thermo-Fisher scientific). Mass spectrometry was performed in a positive ion mode (at mass range of m/z 350-1800 AMU and resolution 70,000) using repetitively full MS scan followed by collision induces dissociation (HCD, at 35 normalized collision energy) of the 10 most dominant ions ( $>1$ charges) selected from the first full MS scan. For the phosphoproteomic profiling, samples underwent phosphorenrichment using Immobilized Metal $(\mathrm{Fe}+3)$ Affinity Chromatography on a robotic system (Bravo) prior to LC-MS/MS analysis.

\section{Mass spectrometry data analyses}

The mass spectrometry data was analyzed using the MaxQuant software 1.5.2.8 (www.maxquant.org, (62)) for peak picking identification and quantitation using the Andromeda search engine, searching against the rat proteome from the Uniprot database with mass tolerance of $20 \mathrm{ppm}$ for the precursor masses and $20 \mathrm{ppm}$ for the fragment ions. Methionine oxidation, phosphorylation (STY) and protein N-terminus acetylation were accepted as variable modifications and carbamidomethyl on cysteine was accepted as static modifications. Minimal peptide length was set to six amino acids and a maximum of two miscleavage events was allowed. Peptide- and protein-level false discovery rates (FDRs) were filtered to $1 \%$ using the target-decoy strategy. The protein table was filtered to eliminate the identifications from the reverse database, and common contaminants. The data was quantified by label free analysis using the MaxQuant software, based on extracted ion currents (XICs) of peptides enabling quantitation from each LC-MS run for each peptide identified in any of experiments.

The rat parathyroid MS proteomics and phosphoproteomics data have been deposited to the ProteomeXchange Consortium via the PRIDE partner repository with the dataset identifiers PXD029368 and PXD029401, respectively (63). For the primary statistical analysis of the MaxQuant proteomic data we uploaded proteinGroups.txt and evidence.txt files to the ProteoSign webserver (64). Supplemental Table S1 and Fig 1 present ProteoSign's differential protein expression results and Supplemental Fig 1 shows ProteoSign's enrichment results, processed with g:Profiler (65) and plotted using ggplot2 (https://ggplot2.tidyverse.org) for R. MaxQuant-identified phosphoproteins, with the detectable proteome as background, subjected to enrichment analysis using DAVID 
(66), and the results displayed with ggplot2. A recently described R package, PhosR, was applied for differential phosphorylation analysis, for phosphosite- and gene-centered enrichment analyses of differentially phosphorylated sites and for kinase perturbation analysis (42). Finally, we searched for significant sequence motif alterations in phosphosites between Adenine and Control with Two Sample Logo approach, using a webserver interface (48).

Cell cultures and transient transfection

HEK293 cells were transiently transfected in 24-well plates for RNA analysis and in 10 $\mathrm{cm}$ plates for protein extractions and IP respectively using a TransFectinTM reagent (Bio-Rad, Hercules, California). $4 \mathrm{~h}$ after transfection, the cells were trypsinized and reseeded in 96 well plates. The next day cells were treated with vehicle (DMSO), forskolin or Sulfopin at $5 \mu \mathrm{M}$, for the indicated time points. RNA was extracted with TRIzol (Invitrogen) for qRT-PCR analysis.

$\underline{\text { Plasmids }}$

The human PTH gene, including exons and introns in pcDNA3 was previously described (53). pCMV-Tag 2B plasmid containing FLAG-full length KHSRP or the KHSRP phosphorylation null mutants Thr692Ala, Thr100Ala where kindly provided by R. Gherzi (IRCCS Ospedale Policlinico San Martino, Genova, Italy). Ser181Ala, Ser181Ala;Thr100Ala double mutant and Ser181Ala;Thr100Ala;Thr692Ala triple mutant were prepared using the QuikChange Lightning Site-Directed Mutagenesis Kit (Agilent Technologies, Santa Clara, CA, US) according to the manufacturer's instructions. The GH expression plasmid was kindly provided by O. Meyuhas (Hebrew University-Hadassah Medical School, Jerusalem, Israel). The GH-PTH mRNA 63nt plasmid (GH63) containing the 63nt rat PTH mRNA ARE cloned between the 3 ' of the $G H$ mRNA coding sequence and the $G H$ mRNA 3' UTR as previously described (11, 54). Empty control vector pcDNA3 (Invitrogen) was used as indicated. GST-Pin1 expression plasmid was a kind gift from K.P. Lu (Beth Israel Deaconess Medical Center, Harvard Medical School, Boston, MA).

Quantitative RT-PCR analysis

cDNA was synthesized using a qScript cDNA Synthesis Kit (Quanta bio, Beverly, MA) and quantitative (q) PCR analysis performed with PerfeCTa SYBR Green FastMix, Low ROX (Quanta bio) in a ViiA 7 Fast Real-Time PCR System (Applied Biosystems Waltham MS). Primers were as follows: mouse PTH: Fw: 5'- 
mouse $\beta$-actin: Fw: 5'-CCTAGGCACCAGGGTGTGAT-3' and Rev: 5'-

TCAGGGTCAGGATACCTCTCTTG-3'; human PTH: Fw: 5'-

CAGATTTCCCATCCGATTTT-3' and Rev:5'-GGGTCTGCAGTCCAATTCAT-3';

human GAPDH: Fw: 5'-TCGGAGTCAACGGATTTG-3' and Rev: 5'-

CAACAATATCCACTTTACCAGAG-3'; human TATA-Box Binding Protein (TBP):

Fw:5'-CACTCACAGACTCTCACAAC-3' and Rev: 5'-

CTGCGGTACAATCCCAGAACT-3'; human GH: Fw: 5'-

CTTATCCAGGCTTTTTGAC-3' and Rev: 5'-TTAAACTCCTGGTAGGTGTC-3'.

Pin1 phosphorylation assay

GST-tagged bait proteins were purified using Pierce GST Protein Interaction Pull-Down

Kit (Thermo-Fisher Scientific). To examine recombinant Pin1 phosphorylation,

parathyroids from control and CKD rats were micro-dissected and homogenized using a

bead beater (a pool from 10 rats in each group) in $80 \mathrm{mM} \beta$-glycerophosphate, $20 \mathrm{mM}$

EGTA, $15 \mathrm{mM} \mathrm{MgCl}_{2}, 50 \mathrm{mM} \mathrm{NaVO}_{4}$, and a protease inhibitor cocktail (Roche). The

lysates were centrifuged for $5 \mathrm{~min}$ at $4^{\circ} \mathrm{C}$ and the kinase assay performed at $37^{\circ} \mathrm{C}$ for 30

min in a $50 \mu \mathrm{l}$ reaction volume containing $50 \mathrm{mM}$ Tris- $\mathrm{HCl}(\mathrm{pH} 7.5), 10 \mathrm{mM} \mathrm{MgCl}, 50$

$\mathrm{mM} \mathrm{KCl}, 1 \mathrm{mM}$ DTT, $1 \mathrm{mM}$ EGTA, $0.16 \mathrm{mCi} / \mathrm{ml}\left[\gamma_{-}{ }^{32} \mathrm{P}\right] \mathrm{ATP}$, parathyroid extracts (10$20 \mu \mathrm{g})$ and GST or GST-Pin1 bound beads $(6 \mu \mathrm{g})$. The beads were then washed and the proteins eluted with reduced glutathione $(10 \mathrm{mM})$. Eluted proteins were run on SDS-

PAGE. ${ }^{32} \mathrm{P}$-labeled GST-Pin1 was detected by autoradiography and GST/GST-Pin1 by Coomassie blue staining of the gels (28). Protein bands were quantified with Quantity One (Bio-Rad).

$\underline{\text { Immunofluorescence staining and quantification }}$

Micro-dissected rat parathyroid glands and mouse thyro-parathyroid tissue were embedded in paraffin and sections prepared. In some experiments, HEK293 cells were grown in 24 well plates. The cells were treated with $5 \mu \mathrm{M}$ forskolin for $1 \mathrm{~h}$ and then fixed with methanol for $5 \mathrm{~min}$. Immunostaining was performed using the following primary antibodies diluted in Cas block (Zymed Laboratories, San Francisco, CA): pPin1 S71 (1:1000, a kind gift from KP Lu, Harvard Boston), p-Pin1 S16 (1:300, BioSS, Woburn, MA), total Pin1 (1:500 Cell Signaling, Beverly, MA), PTH (1:1000, Bio-Rad, Hercules, California). Fluorochrome-conjugated secondary antibodies Cy3 and Cy5 (Bethyl, Montgomery, TX) and nuclear staining SYTOX (Life Technologies) were used for detection. Images were obtained using a Fluoview 1000 Olympus florescence microscope. Immunofluorescence staining was quantified using ImageJ (NIH, Bethesda, 
Maryland). Immunofluorescence staining of HEK293 cells was visualized using the Spinning disk confocal microscopy (SDCM) which improves the speed of image acquisition and NIS elements software (Nikon, Melville, NY) was used for quantification.

Biotinylated RNA protein pull-down assay for Mass Spectrometry was performed at the Weizmann Institute of Science, Rehovot

In vitro transcribed RNA was prepared from linearized plasmid containing the human PTH 3'-UTR (67) using a Biotin RNA Labeling Mix (Roche) and T7 RNA polymerase. Biotinylated RNA protein pull-down was performed according to the protocol of Panda et al (68). Biotinylated RNA was incubated with extracts (1 mg) from HEK293 cells treated with forskolin, Sulfopin or vehicle DMSO, as described above, in triplicate. Nonbiotinylated RNA was transcribed and incubated with cell extracts as background control. Protein-RNA complexes were pulled down using streptavidin-coated beads and eluted with PEB buffer at $100^{\circ} \mathrm{C}$ for $10 \mathrm{~min}$. The samples were analyzed by For liquid chromatography-mass spectrometry (LC-MS). The samples were subjected to tryptic digestion using an S-trap. The resulting peptides were analyzed using Waters HSS-T3 column on nanoflow liquid chromatography (nanoAcquity) coupled to high resolution, high mass accuracy mass spectrometry (Q Exactive Plus). Each sample was analyzed on the instrument separately in a random order in discovery mode. Raw data was processed with MaxQuant v1.6.6.0. The data was searched with the Andromeda search engine against the human proteome database appended with common lab protein contaminants and the following modifications: Fixed modification- cysteine carbamidomethylation. Variable modifications- methionine oxidation, protein N-terminal acetylation, asparagine- or glutamine-deamidation, and serine-, threonine or tyrosinephosphorylation. These MS proteomics data have also been deposited to the ProteomeXchange Consortium with the dataset identifier PXD029456. Data filtration, transformation, normalization, missing value imputation and subsequent differential expression analysis were performed using DEP package (Differential Enrichment analysis of Proteomics data) on R (69). Protein annotations were characterized using DAVID as above. A heat map of differentially-pulled proteins was generated with the NMF package.

\section{Human Parathyroids}

Parathyroid sections from human archival tissue from the Pathology Department of the Hadassah Medical Center were used with approval of the Institutional Helsinki Ethics 
Committee. We studied human parathyroids from ESRD patients that underwent parathyroidectomy for severe SHPT. Control parathyroid glands were incidentally removed during thyroidectomy, performed due to thyroid disease.

$\underline{\text { Statistical analysis }}$

Values are presented as mean \pm SE. A 2-tailed Student's $t$-test was used to assess differences among groups. A $p$ value of less than 0.05 was considered significant.

\section{References}

1. Brown EM, Gamba G, Riccardi D, Lombardi M, Butters R, Kifor O, Sun A, Hediger MA, Lytton J, and Hebert J. Cloning and characterization of an extracellular $\mathrm{Ca}^{2+}$-sensing receptor from bovine parathyroid. Nature. 1993;366(575-80.

2. Wettschureck N, Lee E, Libutti SK, Offermanns S, Robey PG, and Spiegel AM. Parathyroid-specific double knockout of Gq and G11 alpha-subunits leads to a phenotype resembling germline knockout of the extracellular Ca2+-sensing receptor. Mol Endocrinol. 2007;21(1):274-80.

3. Naveh-Many T, Rahamimov R, Livni N, and Silver J. Parathyroid cell proliferation in normal and chronic renal failure rats: the effects of calcium, phosphate and vitamin D. J Clin Invest. 1995;96(1786-93.

4. Naveh-Many T, and Silver J. Regulation of parathyroid hormone gene expression by hypocalcemia, hypercalcemia, and vitamin D in the rat. JClinInvest. 1990;86(1313-9.

5. Slatopolsky E, Lopez-Hilker S, Delmez J, Dusso A, Brown A, and Martin KJ. The parathyroid-calcitriol axis in health and chronic renal failure. Kidney IntSuppl. 1990;29(S41-S7.

6. Lewin E, Garfia B, Recio FL, Rodriguez M, and Olgaard K. Persistent downregulation of calcium-sensing receptor mRNA in rat parathyroids when severe secondary hyperparathyroidism is reversed by an isogenic kidney transplantation. JAmSocNephrol. 2002;13(8):2110-6.

7. Goodman WG, and Quarles LD. Development and progression of secondary hyperparathyroidism in chronic kidney disease: lessons from molecular genetics. Kidney international. 2008;74(3):276-88.

8. Leaf DE, Jacob KA, Srivastava A, Chen ME, Christov M, Jüppner H, Sabbisetti VS, Martin A, Wolf M, and Waikar SS. Fibroblast Growth Factor 23 Levels Associate with AKI and Death in Critical Illness. Journal of the American Society of Nephrology : JASN. 2017;28(6):1877-85.

9. Christov M, Waikar SS, Pereira RC, Havasi A, Leaf DE, Goltzman D, Pajevic PD, Wolf M, and Juppner H. Plasma FGF23 levels increase rapidly after acute kidney injury. Kidney international. 2013;84(776-85.

10. Hassan A, Durlacher K, Silver J, Naveh-Many T, and Levi R. The fibroblast growth factor receptor mediates the increased FGF23 expression in acute and chronic uremia. American Journal of Physiology - Renal Physiology. 2015;310(F217-F21.

11. Nechama M, Ben Dov IZ, Briata P, Gherzi R, and Naveh-Many T. The mRNA decay promoting factor K-homology splicing regulator protein post-transcriptionally determines parathyroid hormone mRNA levels. FASEB J. 2008;22(3458-68.

12. Nechama M, Uchida T, Yosef-Levi IM, Silver J, and Naveh-Many T. The peptidyl-prolyl isomerase Pin1 determines parathyroid hormone mRNA levels and stability in rat models of secondary hyperparathyroidism. The Journal of Clinical Investigation. 2009;119(10):3102-14. 
13. Zhang W, Wagner BJ, Ehrenman K, Schaefer AW, DeMaria CT, Crater D, DeHaven K, Long L, and Brewer G. Purification, characterization, and cDNA cloning of an AU-rich element RNA-binding protein, AUF1. Molecular and cellular biology. 1993;13(12):7652-65.

14. Sela-Brown A, Silver J, Brewer G, and Naveh-Many T. Identification of AUF1 as a parathyroid hormone mRNA 3'-untranslated region binding protein that determines parathyroid hormone mRNA stability. The Journal of biological chemistry. 2000;275(10):7424-9.

15. Wagner BJ, DeMaria CT, Sun Y, Wilson GM, and Brewer G. Structure and genomic organization of the human AUF1 gene: alternative pre-mRNA splicing generates four protein isoforms. Genomics. 1998;48(2):195-202.

16. Gherzi R, Lee KY, Briata P, Wegmuller D, Moroni C, Karin M, and Chen CY. A KH domain RNA binding protein, KSRP, promotes ARE-directed mRNA turnover by recruiting the degradation machinery. MolCell. 2004;14(5):571-83.

17. Briata P, Bordo D, Puppo M, Gorlero F, Rossi M, Perrone-Bizzozero N, and Gherzi R. Diverse roles of the nucleic acid-binding protein KHSRP in cell differentiation and disease. Wiley Interdiscip Rev RNA. 2016;7(2):227-40.

18. Briata P, Forcales SV, Ponassi M, Corte G, Chen CY, Karin M, Puri PL, and Gherzi R. p38-dependent phosphorylation of the mRNA decay-promoting factor KSRP controls the stability of select myogenic transcripts. Mol Cell. 2005;20(6):891-903.

19. Velázquez-Cruz A, Baños-Jaime B, Díaz-Quintana A, De la Rosa MA, and Díaz-Moreno I. Post-translational Control of RNA-Binding Proteins and Disease-Related Dysregulation. Front Mol Biosci. 2021;8(658852-.

20. Murray RD, Merchant ML, Hardin E, Clark B, Khundmiri SJ, and Lederer ED. Identification of an RNA-binding protein that is phosphorylated by PTH and potentially mediates PTH-induced destabilization of Npt2a mRNA. American Journal of Physiology - Cell Physiology. 2016;310(3):C205.

21. Lu KP, Hanes SD, and Hunter T. A human peptidyl-prolyl isomerase essential for regulation of mitosis. Nature. 1996;380(6574):544-7.

22. Innes B, Bailey M, Brandl C, Shilton B, and Litchfield D. Non-catalytic participation of the Pin1 peptidyl-prolyl isomerase domain in target binding. Frontiers in Physiology. 2013;4(18).

23. Lu KP, and Zhou XZ. The prolyl isomerase PIN1: a pivotal new twist in phosphorylation signalling and disease. Nat RevMol Cell Biol. 2007;8(11):904-16.

24. Esnault S, Shen ZJ, and Malter JS. Pinning down signaling in the immune system: the role of the peptidyl-prolyl isomerase Pin1 in immune cell function. Crit Rev/mmunol. 2008;28(1):45-60.

25. Shen ZJ, Esnault S, Schinzel A, Borner C, and Malter JS. The peptidyl-prolyl isomerase Pin1 facilitates cytokine-induced survival of eosinophils by suppressing Bax activation. Nat Immunol. 2009;10(3):257-65.

26. Shen ZJ, and Malter JS. Regulation of AU-Rich Element RNA Binding Proteins by Phosphorylation and the Prolyl Isomerase Pin1. Biomolecules. 2015;5(2):412-34.

27. Krishnan N, Lam TT, Fritz A, Rempinski D, O'Loughlin K, Minderman H, Berezney R, Marzluff WF, and Thapar R. The Prolyl Isomerase Pin1 Targets Stem-Loop Binding Protein (SLBP) To Dissociate the SLBP-Histone mRNA Complex Linking Histone mRNA Decay with SLBP Ubiquitination. Molecular and cellular biology. 2012;32(21):4306-22.

28. Nechama M, Lin CL, and Richter JD. An unusual two-step control of CPEB destruction by Pin1. Molecular and cellular biology. 2013;33(1):48-58.

29. Kumar R. Pin1 regulates parathyroid hormone mRNA stability. J Clin Invest. 2009;119(10):2887-91. 
30. Kilav-Levin R, Hassan A, Nechama M, Shilo V, Silver J, Ben-Dov IZ, and Naveh-Many T. Post-transcriptional mechanisms regulating parathyroid hormone gene expression in secondary hyperparathyroidism. The FEBS Journal. 2020;n/a(n/a).

31. Ranganathan R, Lu KP, Hunter T, and Noel JP. Structural and functional analysis of the mitotic rotamase Pin1 suggests substrate recognition is phosphorylation dependent. Cell. 1997;89(6):875-86.

32. Smet C, Wieruszeski J-M, Buée L, Landrieu I, and Lippens G. Regulation of Pin1 peptidyl-prolyl cis/trans isomerase activity by its WW binding module on a multiphosphorylated peptide of Tau protein. FEBS Letters. 2005;579(19):4159-64.

33. Yaffe MB, Schutkowski M, Shen M, Zhou XZ, Stukenberg PT, Rahfeld JU, Xu J, Kuang J, Kirschner MW, Fischer G, et al. Sequence-specific and phosphorylation-dependent proline isomerization: a potential mitotic regulatory mechanism. Science (New York, NY). 1997;278(5345):1957-60.

34. Lu PJ, Zhou XZ, Liou YC, Noel JP, and Lu KP. Critical Role of WW Domain Phosphorylation in Regulating Phosphoserine Binding Activity and Pin1 Function. Journal of Biological Chemistry. 2002;277(4):2381-4.

35. Lee TH, Chen CH, Suizu F, Huang PY, Schiene-Fischer C, Daum S, Zhang YJ, Goate A, Chen RH, Zhou XZ, et al. Death-Associated Protein Kinase 1 Phosphorylates Pin1 and Inhibits Its Prolyl Isomerase Activity and Cellular Function. Molecular Cell. 2011;42(2):147-59.

36. Liou Y-C, Zhou XZ, and Lu KP. Prolyl isomerase Pin1 as a molecular switch to determine the fate of phosphoproteins. Trends Biochem Sci. 2011;36(10):501-14.

37. Chen $\mathrm{CH}$, Li W, Sultana R, You MH, Kondo A, Shahpasand K, Kim BM, Luo ML, Nechama M, Lin YM, et al. Pin1 cysteine-113 oxidation inhibits its catalytic activity and cellular function in Alzheimer's disease. Neurobiology of disease. 2015;76(13-23.

38. Dubiella C, Pinch BJ, Koikawa K, Zaidman D, Poon E, Manz TD, Nabet B, He S, Resnick $E$, Rogel A, et al. Sulfopin is a covalent inhibitor of Pin1 that blocks Myc-driven tumors in vivo. Nature Chemical Biology. 2021.

39. Kumar $\mathrm{R}$, and Thompson JR. The regulation of parathyroid hormone secretion and synthesis. Journal of the American Society of Nephrology : JASN. 2011;22(2):216-24.

40. Facette MR, Shen Z, Björnsdóttir FR, Briggs SP, and Smith LG. Parallel proteomic and phosphoproteomic analyses of successive stages of maize leaf development. The Plant cell. 2013;25(8):2798-812.

41. Volovelsky O, Cohen G, Kenig A, Wasserman G, Dreazen A, Meyuhas O, Silver J, and Naveh-Many T. Phosphorylation of Ribosomal Protein S6 Mediates Mammalian Target of Rapamycin Complex 1-Induced Parathyroid Cell Proliferation in Secondary Hyperparathyroidism. JAmSocNephrol. 2016;27(4):1091-101.

42. Kim HJ, Kim T, Hoffman NJ, Xiao D, James DE, Humphrey SJ, and Yang P. PhosR enables processing and functional analysis of phosphoproteomic data. Cell reports. 2021;34(8):108771.

43. Abbasian N, Burton JO, Herbert KE, Tregunna BE, Brown JR, Ghaderi-Najafabadi M, Brunskill NJ, Goodall AH, and Bevington A. Hyperphosphatemia, Phosphoprotein Phosphatases, and Microparticle Release in Vascular Endothelial Cells. Journal of the American Society of Nephrology : JASN. 2015;26(9):2152-62.

44. Beck L, and Beck-Cormier S. Extracellular phosphate sensing in mammals: what do we know? Journal of molecular endocrinology. 2020;65(3):R53-r63.

45. Songyang Z, Lu KP, Kwon YT, Tsai LH, Filhol O, Cochet C, Brickey DA, Soderling TR, Bartleson C, Graves DJ, et al. A structural basis for substrate specificities of protein Ser/Thr kinases: primary sequence preference of casein kinases I and II, NIMA, phosphorylase kinase, calmodulin-dependent kinase II, CDK5, and Erk1. Molecular and cellular biology. 1996;16(11):6486-93. 
46. Yeh ES, Lew BO, and Means AR. The loss of PIN1 deregulates cyclin E and sensitizes mouse embryo fibroblasts to genomic instability. The Journal of biological chemistry. 2006;281(1):241-51.

47. Lu M, Berglund E, Larsson C, Höög A, Farnebo LO, and Bränström R. Calmodulin and calmodulin-dependent protein kinase II inhibit hormone secretion in human parathyroid adenoma. The Journal of endocrinology. 2011;208(1):31-9.

48. Vacic V, lakoucheva LM, and Radivojac P. Two Sample Logo: a graphical representation of the differences between two sets of sequence alignments. Bioinformatics (Oxford, England). 2006;22(12):1536-7.

49. Tsiatsiani L, and Heck AJR. Proteomics beyond trypsin. The FEBS Journal. 2015;282(14):2612-26.

50. Durlacher-Betzer K, Hassan A, Levi R, Axelrod J, Silver J, and Naveh-Many T. Interleukin-6 contributes to the increase in fibroblast growth factor 23 expression in acute and chronic kidney disease. Kidney international. 2018;94(2):315-25.

51. Galitzer H, Ben Dov IZ, Silver J, and Naveh-Many T. Parathyroid cell resistance to fibroblast growth factor 23 in secondary hyperparathyroidism of chronic kidney disease. Kidney international. 2010;77(3):211-8.

52. Shilo V, Ben Dov IZ, Nechama M, Silver J, and Naveh-Many T. Parathyroid-specific deletion of dicer-dependent microRNAs abrogates the response of the parathyroid to acute and chronic hypocalcemia and uremia. FASEB Journal. 2015;29(9):3964-76.

53. Galitzer H, Lavi-Moshayoff V, Nechama M, Meir T, Silver J, and Naveh-Many T. The calcium-sensing receptor regulates parathyroid hormone gene expression in transfected HEK293 cells. BMC Biol. 2009;7(17.

54. Kilav R, Silver J, and Naveh-Many T. A conserved cis-acting element in the parathyroid hormone 3 '-untranslated region is sufficient for regulation of RNA stability by calcium and phosphate. JBio/Chem. 2001;276(8727-33.

55. Gherzi R, Chen CY, Ramos A, and Briata P. KSRP controls pleiotropic cellular functions. Seminars in cell \& developmental biology. 2014;34(2-8.

56. Oster M, Reyer H, Gerlinger C, Trakooljul N, Siengdee P, Keiler J, Ponsuksili S, Wolf P, and Wimmers K. mRNA Profiles of Porcine Parathyroid Glands Following Variable Phosphorus Supplies throughout Fetal and Postnatal Life. Biomedicines. 2021;9(5):454.

57. Shoback DM, and Brown EM. Forskolin increases cellular cyclic adenosine monophosphate content and parathyroid hormone release in dispersed bovine parathyroid cells. Metabolism. 1984;33(509-14.

58. Moallem E, Silver J, and Naveh-Many T. Regulation of parathyroid hormone messenger RNA levels by protein kinase $\mathrm{A}$ and $\mathrm{C}$ in bovine parathyroid cells. J Bone Miner Res. 1995;10(447-52.

59. Jia T, Olauson H, Lindberg K, Amin R, Edvardsson K, Lindholm B, Andersson G, Wernerson A, Sabbagh Y, Schiavi S, et al. A novel model of adenine-induced tubulointerstitial nephropathy in mice. Bmc Nephrology. 2013;14(

60. Au - Bi R, Au - Fan Y, Au - Luo E, Au - Yuan Q, and Au - Mannstadt M. Two Techniques to Create Hypoparathyroid Mice: Parathyroidectomy Using GFP Glands and Diphtheria-Toxin-Mediated Parathyroid Ablation. JOVE. 2017121):e55010.

61. Ben Dov IZ, Galitzer H, Lavi-Moshayoff V, Goetz R, M K-o, Mohammadi M, Sirkis R, Naveh-Many T, and Silver J. The parathyroid is a target organ for FGF23 in rats. J Clin Invest. 2007;117(12):4003-8.

62. Tyanova S, Temu T, and Cox J. The MaxQuant computational platform for mass spectrometry-based shotgun proteomics. Nature protocols. 2016;11(12):2301-19.

63. Perez-Riverol Y, Csordas A, Bai J, Bernal-Llinares M, Hewapathirana S, Kundu DJ, Inuganti A, Griss J, Mayer G, Eisenacher M, et al. The PRIDE database and related 
tools and resources in 2019: improving support for quantification data. Nucleic acids research. 2019;47(D1):D442-d50.

64. Theodorakis E, Antonakis AN, Baltsavia I, Pavlopoulos GA, Samiotaki M, Amoutzias GD, Theodosiou T, Acuto O, Efstathiou G, and Iliopoulos I. ProteoSign v2: a faster and evolved user-friendly online tool for statistical analyses of differential proteomics. Nucleic acids research. 2021;49(W1):W573-W7.

65. Raudvere U, Kolberg L, Kuzmin I, Arak T, Adler P, Peterson H, and Vilo J. g:Profiler: a web server for functional enrichment analysis and conversions of gene lists (2019 update). Nucleic acids research. 2019;47(W1):W191-W8.

66. Huang da W, Sherman BT, and Lempicki RA. Systematic and integrative analysis of large gene lists using DAVID bioinformatics resources. Nature protocols. 2009;4(1):4457.

67. Bell O, Silver J, and Naveh-Many T. Identification and characterization of cis-acting elements in the human and bovine parathyroid hormone mRNA 3'-untranslated region. Journal of Bone and Mineral Research. 2005;20(858-66.

68. Panda AC, Martindale JL, and Gorospe M. Affinity Pulldown of Biotinylated RNA for Detection of Protein-RNA Complexes. Bio-protocol. 2016;6(24).

69. Zhang X, Smits AH, van Tilburg GBA, Ovaa H, Huber W, and Vermeulen M. Proteomewide identification of ubiquitin interactions using UbIA-MS. Nature protocols. 2018;13(3):530-50.

\section{Acknowledgements}

We thank R. Gherzi for KSRP (Genova, Italy) plasmids; K.P. Lu (Harvard Boston) for the Pin1 plasmid; the Smoler Proteomics Center (Haifa Technion, Haifa, Israel) and the De Botton Protein Profiling institute of the Nancy and Stephen Grand Israel National Center for Personalized Medicine (Weizmann Institute of Science, Rehovot, Israel) for the MS analysis; G.W. Vainer (Hadassah Hebrew University Medical Center) for helpful discussions and E. Piontek, for professional technical assistance in paraffin embedding and tissue sectioning. This work was supported by grants from the Israel Science Foundation (to T.N-M., ISF 642/16) and the US-Israel Binational Science Foundation (to T.N-M. and I.Z.B-D., BSF 2019300). T.N-M. and M.N. are research associates of the Wohl's Translation Research Institute at Hadassah-Hebrew University Medical Center. N.L. is the incumbent of the Alan and Laraine Fischer Career Development Chair and is supported by the Estate of Emile Mimran, Honey and Dr. Barry Sherman Lab, Dr. Barry Sherman Institute for Medicinal Chemistry, and Nelson P. Sirotsky.

\section{Figure Legends}

\section{Figure 1. Chronic kidney disease leads to proteomic changes in parathyroid tissue.}

Male rats were fed either a control or an adenine-rich high phosphorus diet for 2 wk to induce CKD and extracts were prepared from micro-dissected parathyroid glands for MS 
proteome analysis. (A) Principal component analysis (PCA) plot of mass spectrometry intensity data ( $\mathrm{n}=4950$ protein groups) showing proteomics-based distinction of adenine from control glands. (B) Volcano plot showing the landscape of protein dysregulation in adenine glands. (C) Enrichment of Reactome pathway-associated proteins among the differentially expressed parathyroid proteins (see also Figure S1).

\section{Figure 2. Chronic kidney disease leads to phosphoproteomic changes in}

parathyroid tissue. (A) Volcano plot showing phosphosite dysregulation in parathyroid extracts from rats fed adenine-rich ( 2 pools of $x$ rats) versus control diet ( 2 pools). The top upregulated phosphosites (log2 fold change $\geq 5$ ) were Sptbn1:S2099, Bckdk:T32, Hist3h2ba:S5, Tmem69:S13, Hp1bp3:T51 and Virma:S1578, while Casr:S1066, Evl:S333, Lmo7:S619, Lpp:T630, Krt86:S47 and Ptbp1:S140 were the most downregulated sites. (B) Two sample logo (48) presenting the only statistically robust sequence difference between phosphosites increased in adenine rats and phosphosites decreased in adenine rats; the odds ratio for $\mathrm{P}$ in the 9 th position in adenine vs. control was 0.79 , Bonferroni-corrected $\mathrm{p}<0.0001$. The inset shows additional differences for which the Bonferroni-corrected p-values were insignificant. (C) KSRP phosphorylation sites detected by mass spectrometry analysis as above. Peptides that are potential target sites for Pin1, Ser182 (VQIS, valine-glutamine-isoleucine-serine-proline), Thr101 (VNNNT, valine-asparagine-asparagine-asparagine-threonine-proline) were increased and Ser481 (LFVIRGSP, leucine-phenylalanine-valine-isoleucine-arginine-glycineserine-proline) was decreased in parathyroids obtained from adenine (CKD) rats.

Figure 3. Chronic kidney disease leads to increased parathyroid Pin1 Ser16 and Ser 71 phosphorylation. (A) In vitro phosphorylation assay of recombinant GST-Pin1 by parathyroid extracts from control $(\mathrm{C})$ and chronic kidney failure (CKD) rats. Rats were fed either an adenine-rich high phosphorus or a control diet for $2 \mathrm{wk}$ to induce CKD. Extracts from pools of 11-13 rats in each group were incubated with GST or GST-Pin1 and $\left[\gamma^{32} \mathrm{P}\right]$ ATP. ${ }^{32} \mathrm{P}$-labeled GST-Pin1 was detected by SDS PAGE autoradiography (top) and Coomassie blue-staining for input GST and GST-Pin1 recombinant proteins (bottom). (B) Quantification of the intensity in autographs as in A and data from additional 3 repeat experiments, presented as fold change compared to control. (C) Schematic representation of Pin1 protein showing the WW protein binding and the PPIase catalytic (PPIase) domains. The regulatory Ser16 and Ser71 phosphorylation sites and their respective kinases as well as the Cys113 site and modulators are shown. (D-G). 
Pin1 immunofluorescent staining of thyroparathyroid sections from control and CKD rats as in A. Sections were stained for SYTOX nuclear staining (green) and antibodies for PTH (blue), Pin1 p-Ser16 (D) and p-Ser71 (B) (red) and merge (purple). (E\&G). Quantification of relative fluorescence intensity for Pin1 in slides as in D and F and additional rats, measured by Image $\mathbf{J}$ software. (H-K) Pin1 immunofluorescent staining of thyroparathyroid sections from control and folic acid induced acute kidney injury (AKI) mice at $24 \mathrm{~h}(\mathbf{H})$ or $48 \mathrm{~h}(\mathbf{J})$ post folic acid or vehicle (control) injection. Sections were stained as above with Pin1 p-Ser16 (H) or p-Ser71 (J). (I\&K) Quantification of Pin1 staining as in $\mathrm{H}$ and $\mathrm{J}$ and additional mouse samples. Results in E,G,I,K are presented as mean $\pm \mathrm{SE}$ of fold change, compared to glands from controls. ${ }^{*}, \mathrm{p}<0.05$.

Figure 4. PKA induced Ser16 phosphorylation at the Pin1 WW domain increases PTH expression in mouse parathyroid glands in culture and in transfected HEK293 cells. (A) Mouse thyro-parathyroid glands were microdissected and the two glands form each mouse maintained in culture with the PKA activator forskolin $(100 \mu \mathrm{M})$ or vehicle (DMSO). PTH accumulated in the culture medium was measured at 1 and 3 h. (B) Rat micro-dissected parathyroid glands (two glands form each rat) were maintained in culture with the PKA inhibitor H89 $(150 \mu \mathrm{M})$ or vehicle (DMSO) and secreted rat PTH measure at $24 \mathrm{~h}$. (C) Mice were fed a normal (Control) or an adenine-rich high phosphorus diet to induce CKD. At 2 wk, thyro-parathyroid glands were cultured with H89 or DMSO (+D) as in B. PTH accumulated in the culture medium was measured at 7 and $24 \mathrm{~h}$. (D) Mice received a single injection of folic acid $(240 \mathrm{mg} / \mathrm{kg})$ to induce acute kidney injury (AKI). At $24 \mathrm{~h}$ thyro-parathyroid glands were removed and cultured with H89 or DMSO (+D) and PTH accumulated in the culture medium measured. (E) HEK293 cells were transiently transfected with an expression plasmid for the human PTH gene. Transfected cells were incubated with forskolin $(10 \mu \mathrm{M})$ or DMSO. PTH mRNA levels at 3 and $24 \mathrm{~h}$, normalized to GAPDH, were determined by qRT-PCR. Results are presented as mean \pm SE of accumulated PTH (A-D) or fold change of PTH mRNA (E), compared to vehicle treated glands or cells. *, p<0.05 compared to control animals or cells incubated with DMSO; \#, $\mathrm{p}<0.05$ compared to control+D.

Figure 5. Targeting Pin1 Cys113 at the catalytic domain by the covalent Pin1 inhibitor sulfopin increases PTH expression in mouse parathyroid glands in culture. (A) The exposed neck area in a mouse that shows YFP expression specifically in the parathyroids (PT-YFP) used for fluorescence-guided micro-surgery of the parathyroid glands with minimal contamination of the adjacent thyroid tissue. PT, 
parathyroid, T, thyroid, Tr, trachea. (B) Microdissected parathyroid glands from PT-YFP mice were maintained in culture with Sulfopin $(50 \mu \mathrm{M})$ or DMSO and PTH accumulated in the growth medium measured at $24 \mathrm{~h}$. Results are presented as mean $\pm \mathrm{SE}$, compared vehicle treated glands. *, $\mathrm{p}<0.05$. (C) PTH mRNA levels normalized to $\beta$-actin were determined by qRT-PCR in pooled glands from $(\mathbf{B})$ and an additional repeat experiment, each composed of pooled parathyroid glands from 5 mice in each group.

Figure 6. Pin1 inhibition by forskolin or sulfopin increases PTH mRNA levels through the PTH mRNA 3'-UTR 63 nt protein binding element. (A) Schematic representation of the constructs used for transfection, showing the $\mathrm{PTH}$, native growth hormone (GH) and the reporter GH containing the PTH 3'-UTR AU rich 63 nt cis acting element (ARE) mRNAs (GH63). CDS, coding sequence; UTR, un-translated region. (BC) HEK293 cells were transiently transfected with expression plasmids for GH or GH63 (GH63). Cells were treated with Forskolin $(5 \mu \mathrm{M})(\mathbf{B})$ or Sulfopin $(5 \mu \mathrm{M})(\mathbf{C})$ or vehicle (DMSO, +D). PTH mRNA levels were measures at $24 \mathrm{~h}$ by qRT-PCR, normalized to TATA box binding protein (TBP). Results are presented as mean $\pm \mathrm{SE}$ of fold change, compared to GH mRNA in vehicle treated cells. *, p<0.05 inhibitor vs vehicle.

Figure 7. Mass-spectrometry based discovery of PTH mRNA binding proteins and the response to Pin1 inhibition mimicking CKD. (A) Characteristics of the PTH 3'UTR specifically bound (pulled-down) proteins when evaluated for enrichment in Reactome database pathways at an adjusted p-value cutoff 0.01. Supplemental Fig 5B expands this analysis to additional data sources. (B) Results of differential pulldown analysis from Pin1-inhibited cell extracts versus control cells, wherein the y-axis shows the effect of Sulfopin and the x-axis shows the effect of forskolin. (C) Heat map showing intensity values of the same set of differentially-pulled-down proteins.

Figure 8. Pin1 induced changes in KSRP phosphorylation alter PTH expression. (A) Schematic representation of the primary structure of KSRP, including the four hnRNP K homology $(\mathrm{KH})$ domains and position of the relevant phosphorylation sites. (B-D) HEK293 cells were transiently transfected with expression plasmids for human PTH without and with native KSRP or KSRP phosphorylation null single mutants Thr100Ala (T100A) (A); Thr692Ala (T692A) or Ser181Ala S181A) (B); KSRP

Ser181Ala;Thr100Ala (double) or Ser181Ala;Thr100Ala;Thr692Ala (triple) mutants (C). Transfected cells were incubated with forskolin (FK) or vehicle DMSO (+D). PTH mRNA levels at $24 \mathrm{~h}$, normalized to GAPDH, were determined by qRT-PCR. Results are 
presented as mean $\pm \mathrm{SE}$ of fold change, compared cells transfected with $\mathrm{PTH}$ expression plasmid and a control empty vector. *, p<0.05 forskolin vs DMSO; \#, vs KSRP+D.

Fig 9. Pin1 phosphorylation in human parathyroid sections from control and end stage renal disease patients. Representative parathyroid sections from end stage renal disease (ESRD) patients and control parathyorids removed at total thyroidectomy were stained by immunofluorescence for sytox nuclear staining (green) and antibodies for PTH (blue), Pin1 p-Ser16 (A) or p-Ser71 (B) (red) and merge (purple). Similar results were obtained in additional 3 control and CKD patients for pSer16 and 2 control and 3 CKD patients for pSer71.

Figure 10. Model for the changes induced by CKD in the parathyroid cells that lead to increased PTH gene expression in SHP. Under basal conditions, Pin1 is unphosphorylated at Ser16 and 71 and thus active, leading to conformational change induced decrease in KSRP phosphorylation. Unphosphorylated KSRP binds to the adenine uridine rich element (ARE) in the PTH mRNA 3'-untranslated region and along with other PTH mRNA binding proteins determines steady state PTH levels. CKD induces Pin1 phosphorylation and hence decreased isomerase activity, leaving KSRP phosphorylated at three Pin1 target sites. Phosphorylated KSRP fails to bind PTH mRNA and induce its degradation, resulting in increased PTH mRNA and serum levels. 

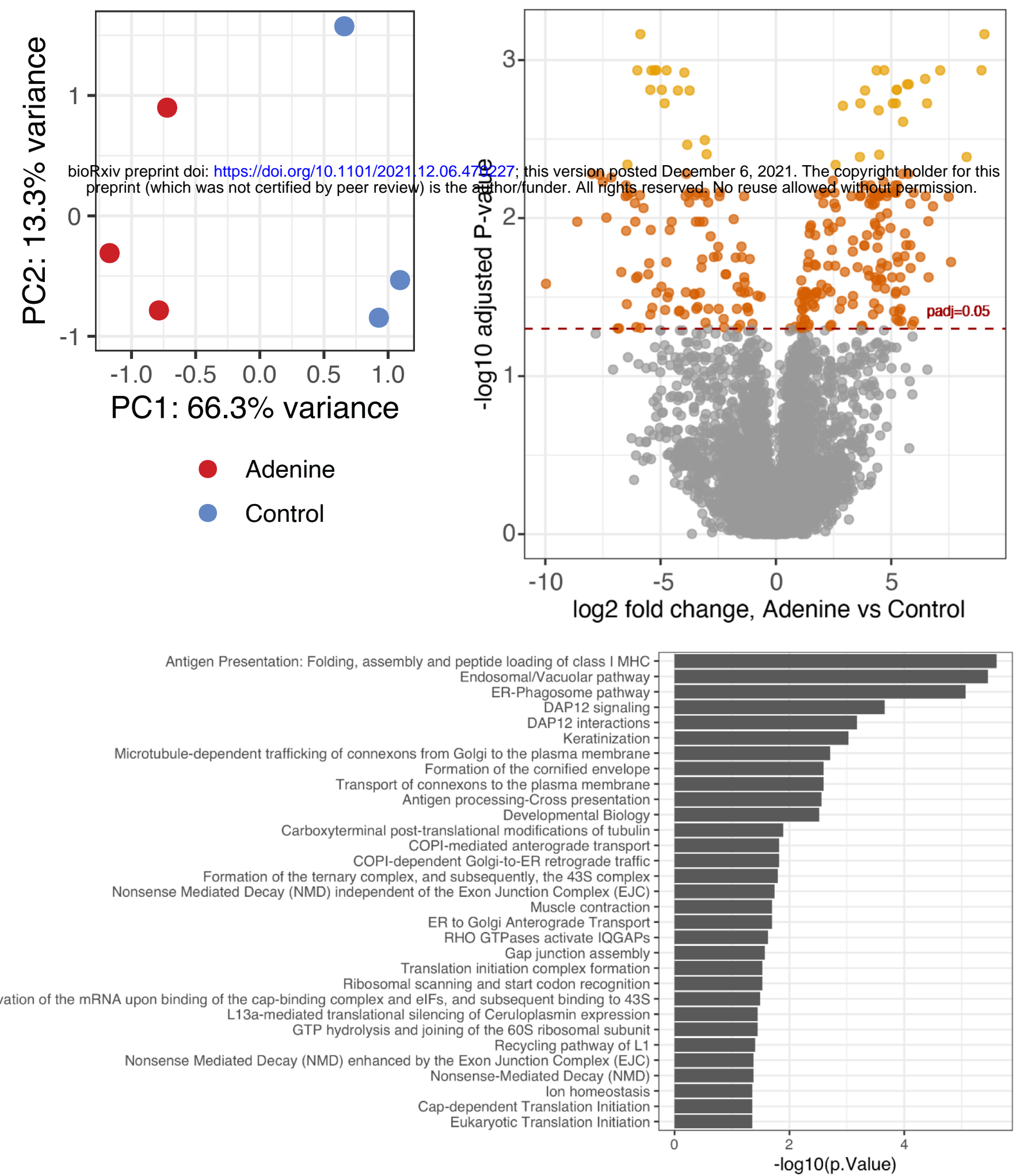

Figure 1. Chronic kidney disease leads to proteomic changes in parathyroid tissue. Rats were fed either a control or an adenine-rich high phosphorus diet for 2 wk to induce CKD and extracts were prepared from micro-dissected parathyroid glands for MS proteome analysis. (A) Principal component analysis (PCA) plot of mass spectrometry intensity data ( $\mathrm{n}=4950$ protein groups) showing proteomics-based distinction of adenine from control glands. (B) Volcano plot showing the landscape of protein dysregulation in adenine glands. (C) Enrichment of Reactome pathwayassociated proteins among the differentially expressed parathyroid proteins (see also Figure S1). 


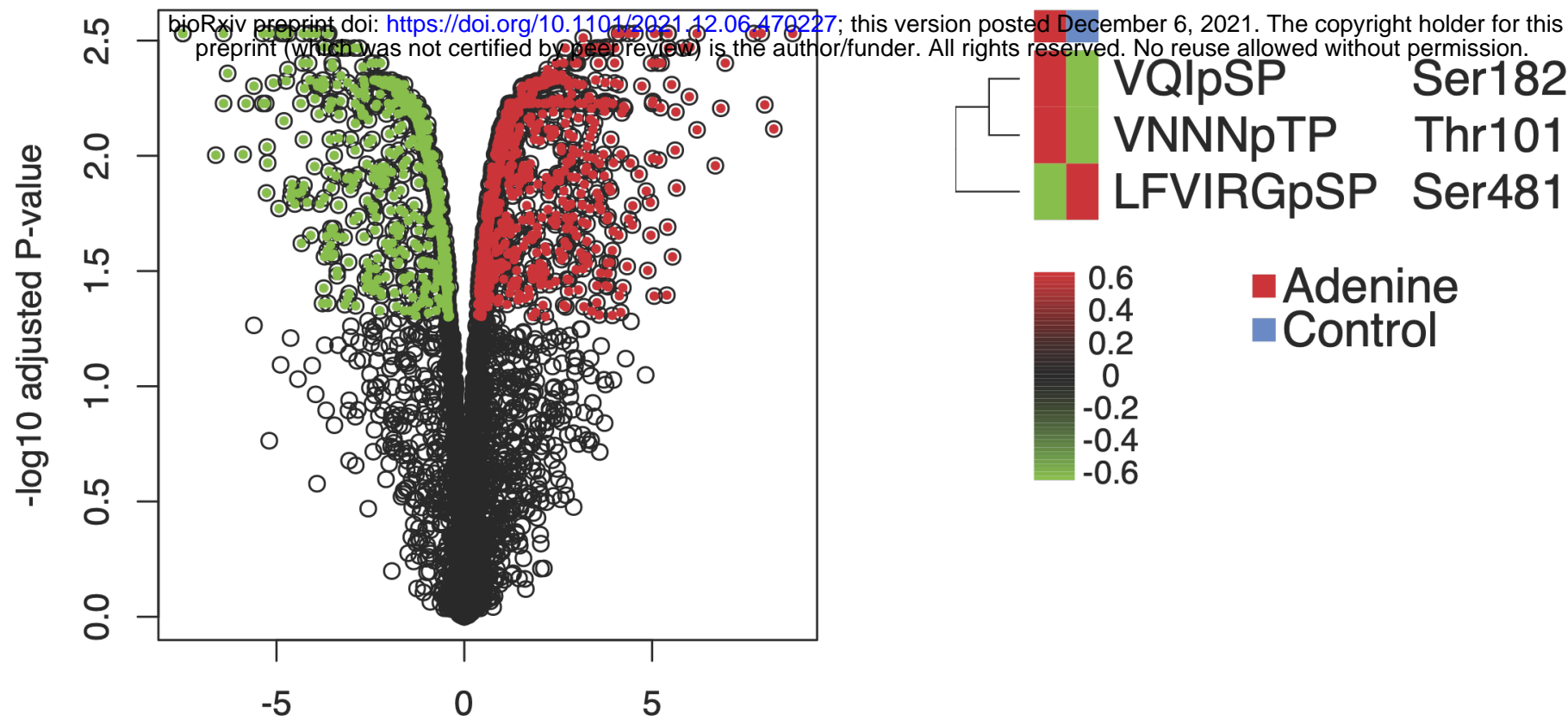

log2 fold-change, Adenine versus Control

B

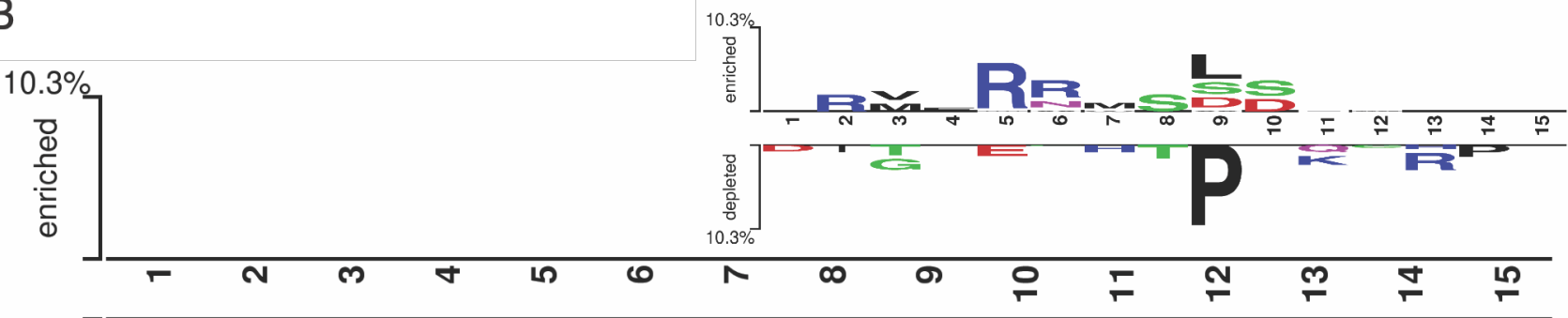

$$
\frac{\frac{9}{2}}{\frac{0}{0}}
$$
$10.3 \%$

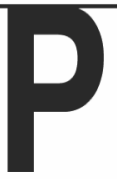

Figure 2. Chronic kidney disease leads to phospho-proteomic changes in parathyroid tissue. (A) Volcano plot showing phosphosite dysregulation in parathyroid extracts from rats fed adenine-rich (2 pools of 6 rats) versus control diet ( 2 pools). The top upregulated phosphosites ( $\log 2$ fold change $\geq 5$ ) were Sptbn1:S2099, Bckdk:T32, Hist3h2ba:S5, Tmem69:S13, Hp1bp3:T51 and Virma:S1578, while Casr:S1066, Evl:S333, Lmo7:S619, Lpp:T630, Krt86:S47 and Ptbp1:S140 were the most downregulated sites. (B) Two sample (48) presenting the only statistically robust sequence difference between phosphosites increased in adenine rats and phosphosites decreased in adenine rats; the odds ratio for $\mathrm{P}$ in the 9 th position in adenine vs. control was 0.79 , Bonferroni-corrected $\mathrm{p}<0.0001$. The inset shows additional differences for which the Bonferroni-corrected p-values were insignificant. (C) KSRP phosphorylation sites detected by mass spectrometry analysis as above. Peptides that are potential target sites for Pin1, Ser182 (VQIS, valine-glutamine-isoleucine-serine-proline), Thr101 (VNNNT, valineasparagine-asparagine-asparagine-threonine-proline) were increased and Ser481 (LFVIRGSP, leucinephenylalanine-valine-isoleucine-arginine-glycine-serine-proline) was decreased in parathyroids obtained from adenine $(\mathrm{CKD})$ rats. 


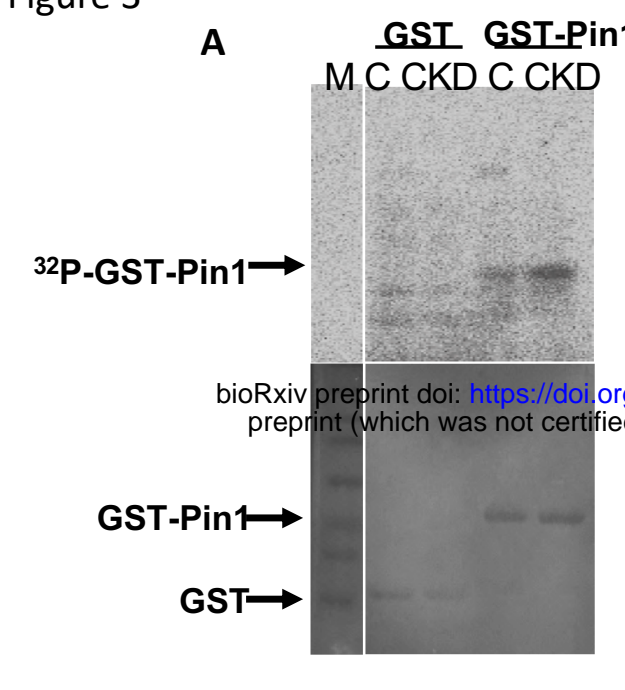

B

C

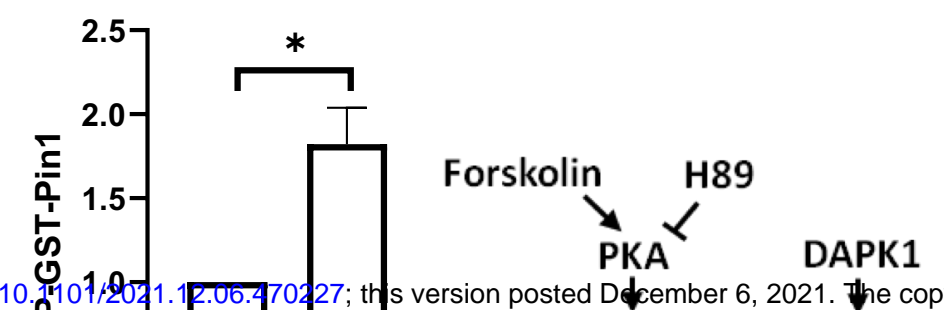

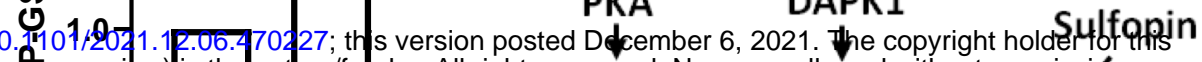
cons

D
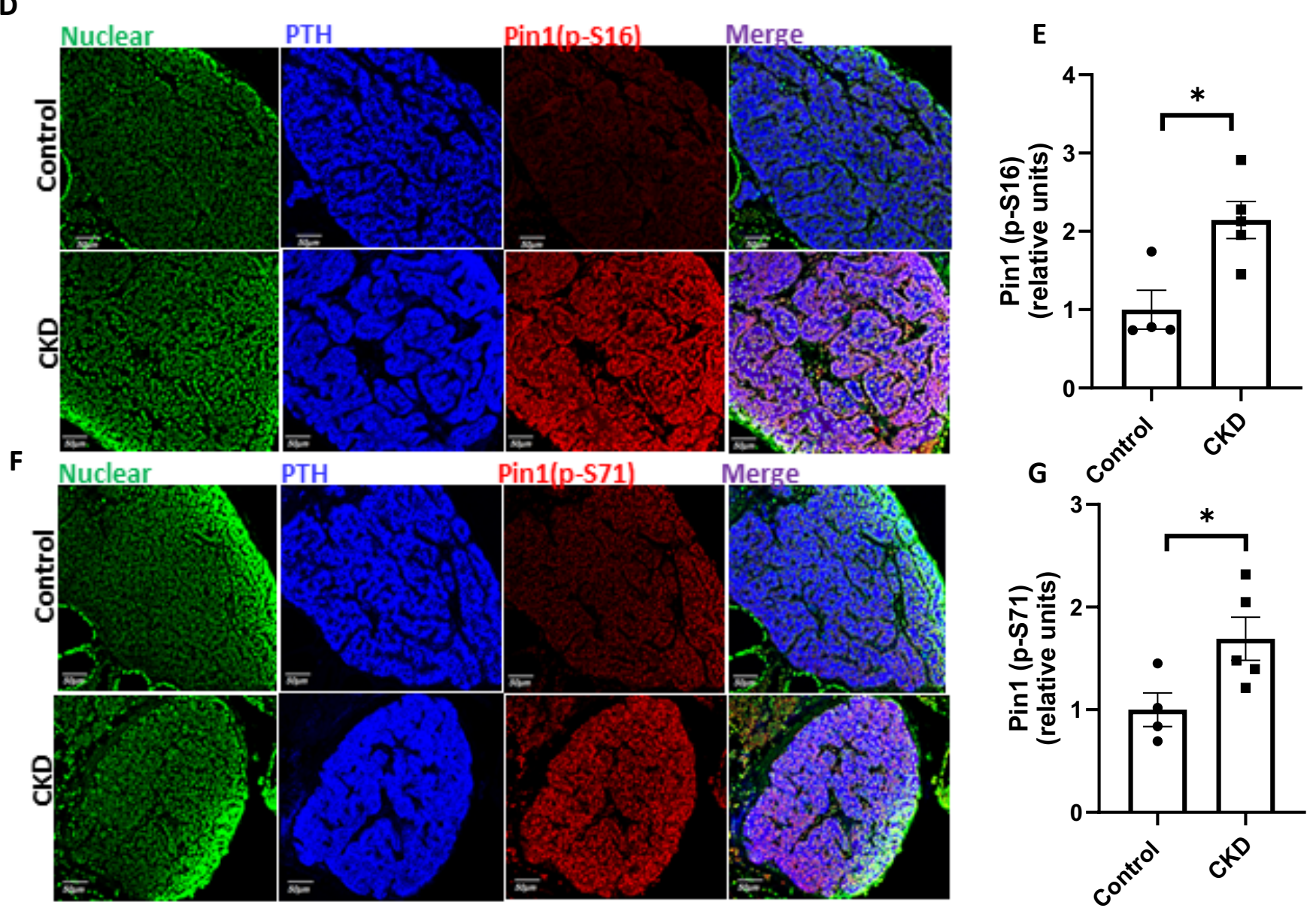

H
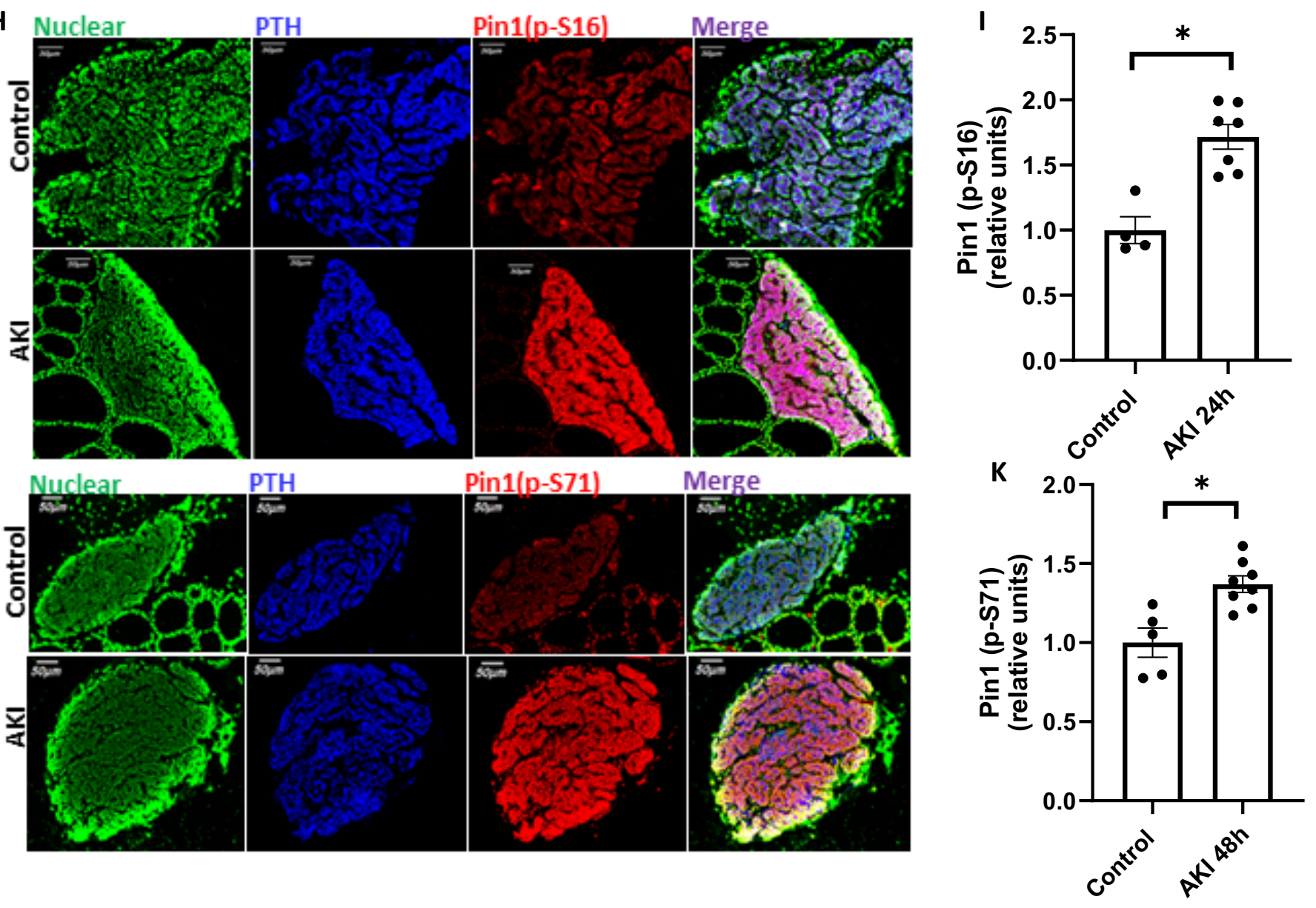
Figure 3. Chronic kidney disease leads to increased parathyroid Pin1 Ser16 and Ser 71

phosphorylation. (A) In vitro phosphorylation assay of recombinant GST-Pin1 by parathyroid extracts from control (C) and chronic kidney failure (CKD) rats. Male rats were fed either an adenine-rich high phosphorus or a control diet for $2 \mathrm{wk}$ to induce CKD. Extracts from pools of 11-13 rats in each group were incubated with GST or GST-Pin1 and $\left[\gamma^{32} \mathrm{P}\right]$ ATP. ${ }^{32} \mathrm{P}-$-labeled GST-Pin1 was detected by SDS PAGE autoradiography (top) and Coomassie blue-staining for input GST and GST-Pin1 recombinant proteins (bottom). (B) Quantification of the intensity in autographs as in A and data from additional 3 repeat experiments, presented as fold change

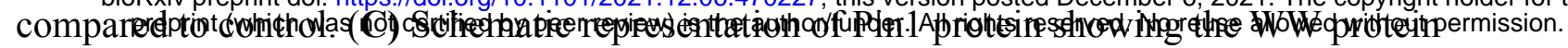
binding and the PPIase catalytic (PPIase) domains. The regulatory Ser16 and Ser71 phosphorylation sites and their respective kinases as well as the Cys113 site and modulators are shown. (D-G). Pin1 immunofluorescent staining of thyroparathyroid sections from control and CKD rats as in A. Sections were stained for SYTOX nuclear staining (green) and antibodies for PTH (blue), Pin1 p-Ser16 (D) and p-Ser71 (B) (red) and merge (purple). (E\&G). Quantification of relative fluorescence intensity for Pin 1 in slides as in D and F and additional rats, measured by Image $\mathbf{J}$ software. (H-K) Pin1 immunofluorescent staining of thyroparathyroid sections from control and folic acid induced acute kidney injury (AKI) mice at $24 \mathrm{~h}(\mathbf{H})$ or $48 \mathrm{~h}(\mathbf{J})$ post folic acid or vehicle (control) injection. Sections were stained as above with Pin1 p-Ser16 (H) or p-Ser71 (J). (I\&K) Quantification of Pin1 staining as in H and $\mathrm{J}$ and additional mouse samples. Results in E,G,I,K are presented as mean $\pm \mathrm{SE}$ of fold change, compared to glands from controls. *, $\mathrm{p}<0.05$. 
Figure 4

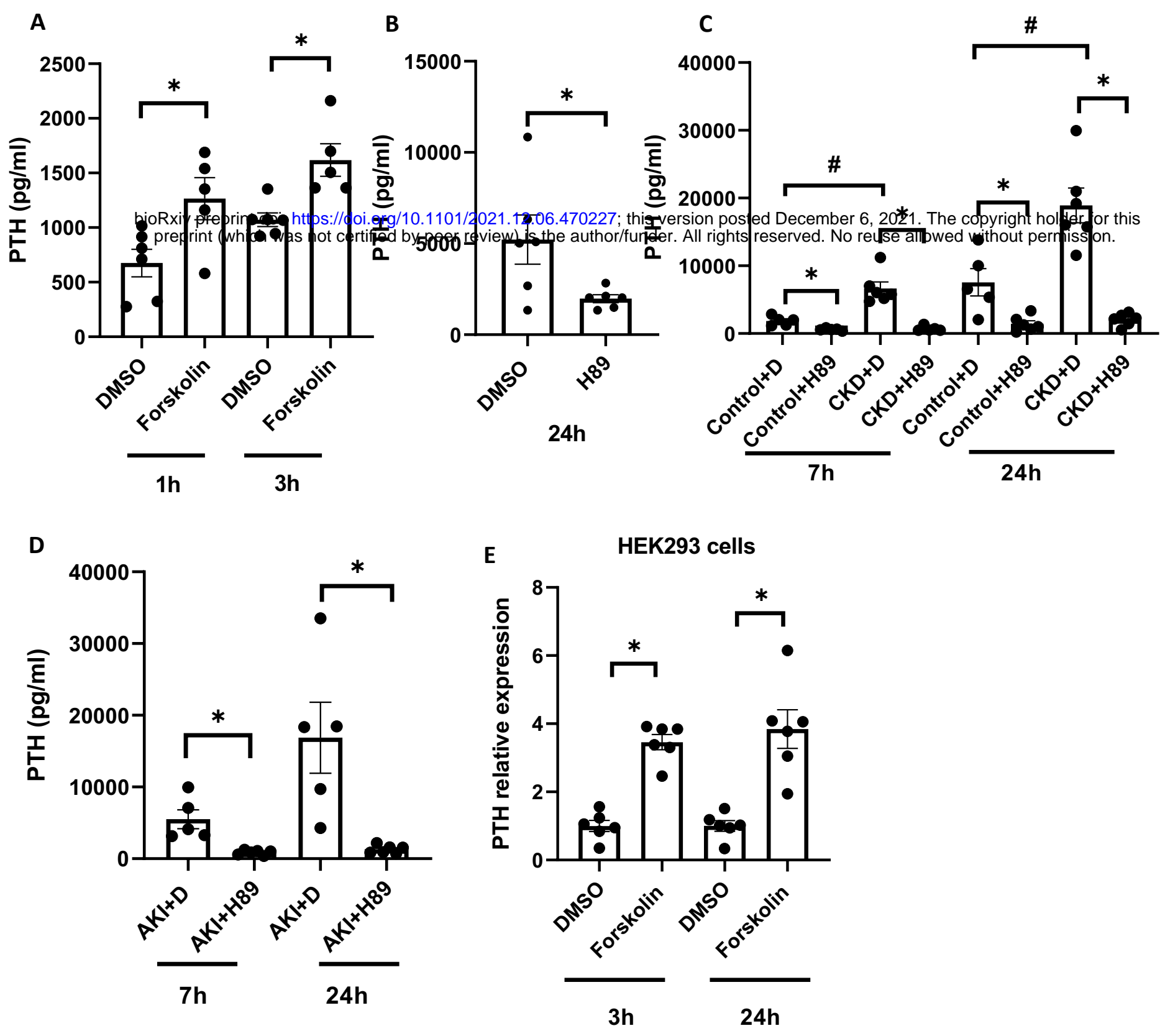

Figure 4. PKA induced Ser16 phosphorylation at the Pin1 WW domain increases PTH expression in mouse parathyroid glands in culture and in transfected HEK293 cells. (A) Mouse thyro-parathyroid glands were microdissected and the two glands form each mouse maintained in culture with the PKA activator forskolin $(100 \mu \mathrm{M})$ or vehicle (DMSO). PTH accumulated in the culture medium was measured at 1 and $3 \mathrm{~h}$. (B) Rat micro-dissected parathyroid glands (two glands form each rat) were maintained in culture with the PKA inhibitor H89 (150 $\mu \mathrm{M})$ or vehicle (DMSO) and secreted rat PTH measure at $24 \mathrm{~h}$. (C) Mice were fed a normal (Control) or an adenine-rich high phosphorus diet to induce CKD. At $2 \mathrm{wk}$, thyro-parathyroid glands were cultured with $\mathrm{H} 89$ or DMSO (+D) as in B. PTH accumulated in the culture medium was measured at 7 and $24 \mathrm{~h}$. (D) Mice received a single injection of folic acid $(240 \mathrm{mg} / \mathrm{kg})$ to induce acute kidney injury (AKI). At $24 \mathrm{~h}$ thyro-parathyroid glands were removed and cultured with H89 or DMSO (+D) and PTH accumulated in the culture medium measured. (E) HEK293 cells were transiently transfected with an expression plasmid for the human PTH gene. Transfected cells were incubated with forskolin $(10 \mu \mathrm{M})$ or DMSO. PTH mRNA levels at 3 and $24 \mathrm{~h}$, normalized to GAPDH, were determined by qRT-PCR. Results are presented as mean \pm SE of accumulated PTH (A-D) or fold change of PTH mRNA (E), compared to vehicle treated glands or cells. ${ }^{*}, \mathrm{p}<0.05$ compared to control animals or cells incubated with DMSO; \#, $\mathrm{p}<0.05$ compared to control+D. 
A

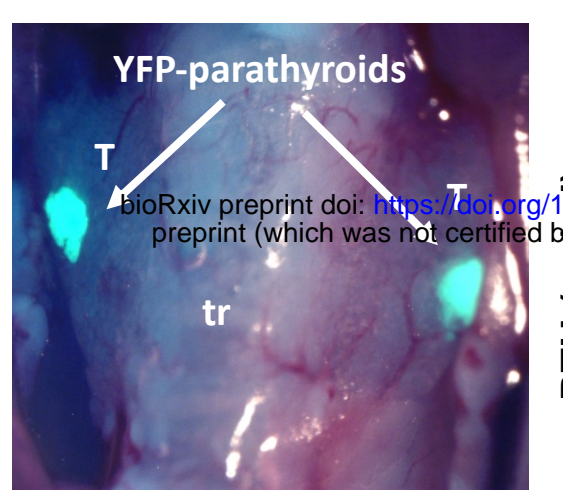

B

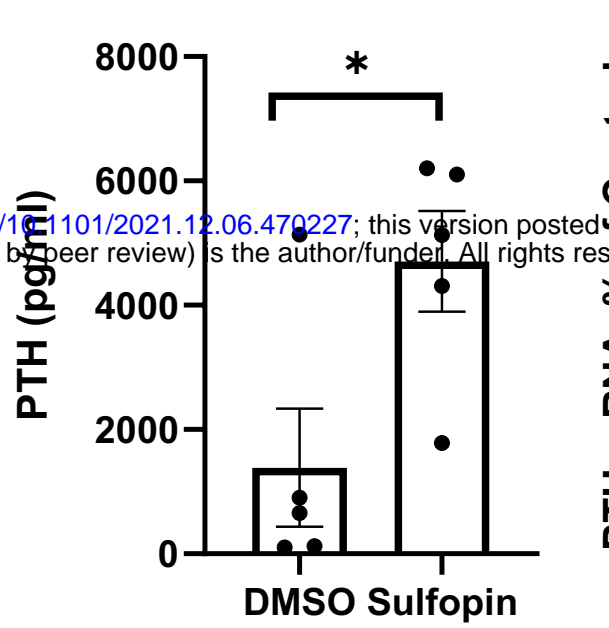

C

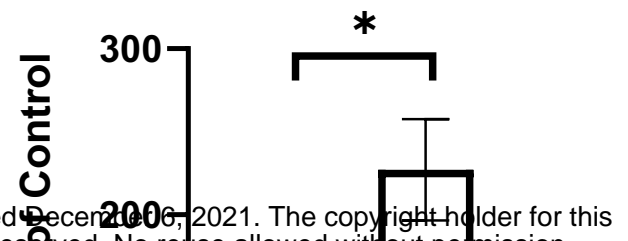

Qved. No reuse allowed with out pe mission.

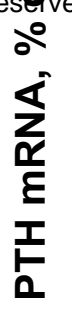$$
\text { No resse allowed with out pe mission. }
$$

Figure 5. Targeting Pin1 Cys113 at the catalytic domain by the covalent Pin1 inhibitor sulfopin increases PTH expression in mouse parathyroid glands in culture. (A) The exposed neck area in a mouse that shows YFP expression specifically in the parathyroids (PT-YFP) used for fluorescenceguided micro-surgery of the parathyroid glands with minimal contamination of the adjacent thyroid tissue. PT, parathyroid, T, thyroid, Tr, trachea. (B) Microdissected parathyroid glands from PT-YFP mice were maintained in culture with Sulfopin $(50 \mu \mathrm{M})$ or DMSO and PTH accumulated in the growth medium measured at $24 \mathrm{~h}$. Results are presented as mean $\pm \mathrm{SE}$, compared vehicle treated glands. *, $\mathrm{p}<0.05$. (C) PTH mRNA levels normalized to $\beta$-actin were determined by qRT-PCR in pooled glands from $\mathbf{B}$ and an additional repeat experiment, each composed of pooled parathyroid glands from 5 mice in each group. 
A

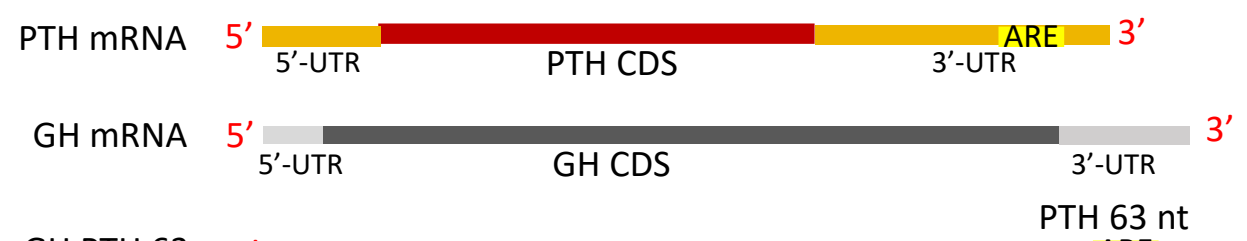

GH PTH 63 6 biokiv preprint doi: https://doi.org/10.1101/2021.12.06.470227; this version posted December 6, ${ }^{\prime}{ }^{\prime} 21$. The copyright holder for this preprint (which was not certified by peer review) is the author/funder. All rights reserved. No reuse allowed without permission.

B

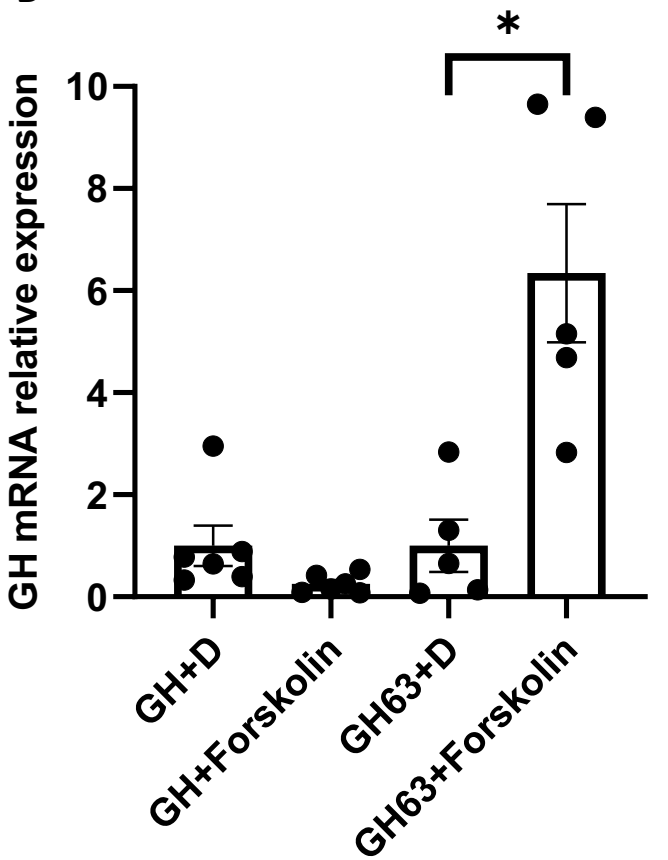

C

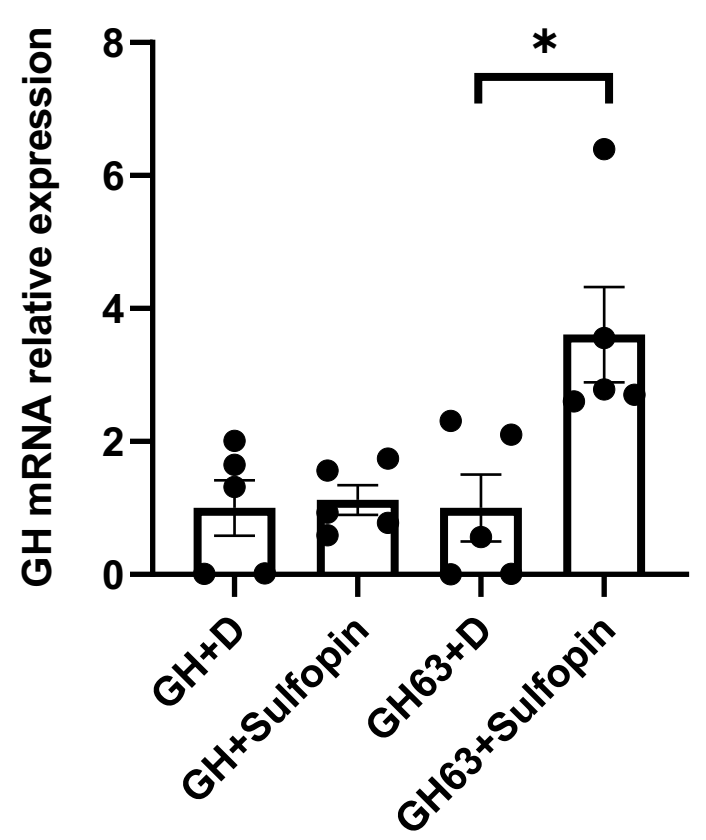

Figure 6. Pin1 inhibition by forskolin or sulfopin increases PTH mRNA levels through the PTH mRNA 3'-UTR 63 nt protein binding element. (A) Schematic representation of the constructs used for transfection, showing the PTH, native growth hormone $(\mathrm{GH})$ and the reporter GH containing the PTH 3'-UTR AU rich 63 nt cis acting element (ARE) mRNAs (GH63). CDS, coding sequence; UTR, un-translated region. (B-C) HEK293 cells were transiently transfected with expression plasmids for GH or GH63 (GH63). Cells were treated with Forskolin $(5 \mu \mathrm{M})(\mathbf{B})$ or Sulfopin $(5 \mu \mathrm{M})(\mathbf{C})$ or vehicle (DMSO, $+\mathrm{D})$. PTH mRNA levels were measures at $24 \mathrm{~h}$ by qRT-PCR, normalized to TATA box binding protein (TBP). Results are presented as mean \pm SE of fold change, compared to GH mRNA in vehicle treated cells. ${ }^{*}, \mathrm{p}<0.05$ inhibitor vs vehicle. 
A

$$
\begin{array}{r}
\text { Metabolism of RNA } \\
\text { mRNA Splicing - Minor Pathway } \\
\text { mRNA Splicing - Major Pathway } \\
\text { mRNA Splicing - }
\end{array}
$$

Processing of Capped Intron-Containing Pre-mRNA -

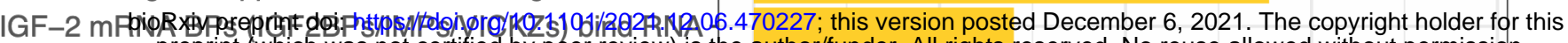
preprint (which was not certified by peer review) is the author/funder. All rights reserved. No reuse allowed without permission.
DNA Replication Pre-Initiation -

Regulation of expression of SLITs and ROBOs -

Small interfering RNA (siRNA) biogenesisCellular responses to stress -

Synthesis of DNA -

Cellular responses to external stimuli DNA Replication G1/S TransitionSignaling by ROBO receptors MAPK6/MAPK4 signaling

Mitotic $\mathrm{G} 1$ phase and $\mathrm{G} 1 / \mathrm{S}$ transition Regulation of HSF1-mediated heat shock responseMetabolism of amino acids and derivatives MicroRNA (miRNA) biogenesis -
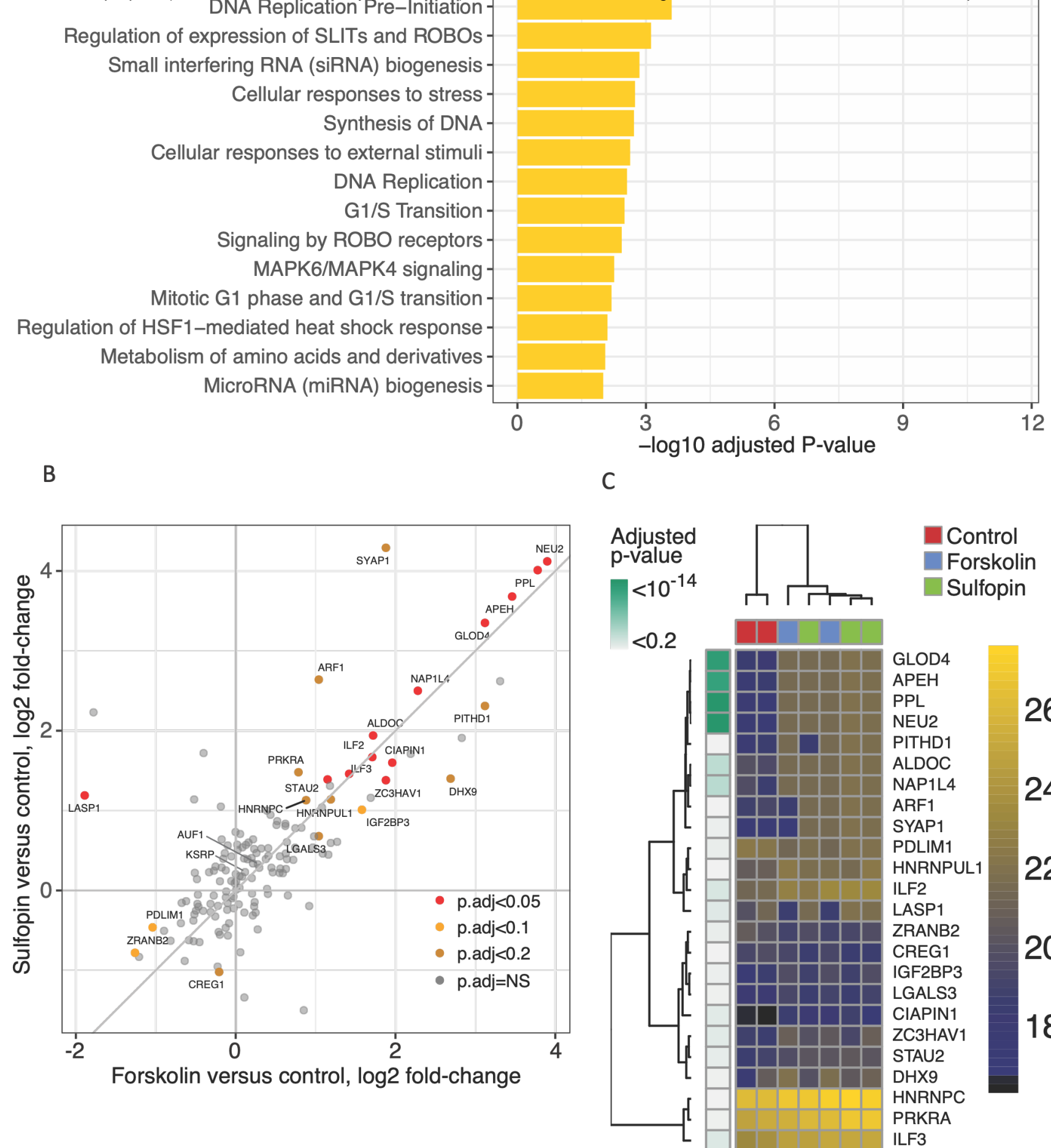

Control

Forskolin

Sulfopin

$<0.2$

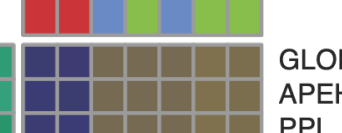

PPL

NEU2

ALDOC

NAP1L

NAP1

ARF1

SYAP1

PDLIM1

HNRNPUL1

F2

ZRANB2

CREG1

IGF2BP3

LGALS3

CIAPIN1

ZC3HAV1

STAU2

DHX9

HNRNPC

PRKRA

ILF3

Figure 7. Mass-spectrometry based discovery of PTH mRNA binding proteins and the response to Pin1 inhibition mimicking CKD. (A) Characteristics of the PTH 3'-UTR specifically bound (pulleddown) proteins when evaluated for enrichment in Reactome database pathways at an adjusted p-value cutoff 0.01. Fig S5B expands this analysis to additional data sources. (B) Results of differential pulldown analysis from Pin1-inhibited cell extracts versus control cells, wherein the y-axis shows the effect of Sulfopin and the x-axis shows the effect of forskolin. (C) Heat map showing intensity values of the same set of differentially-pulled-down proteins. 


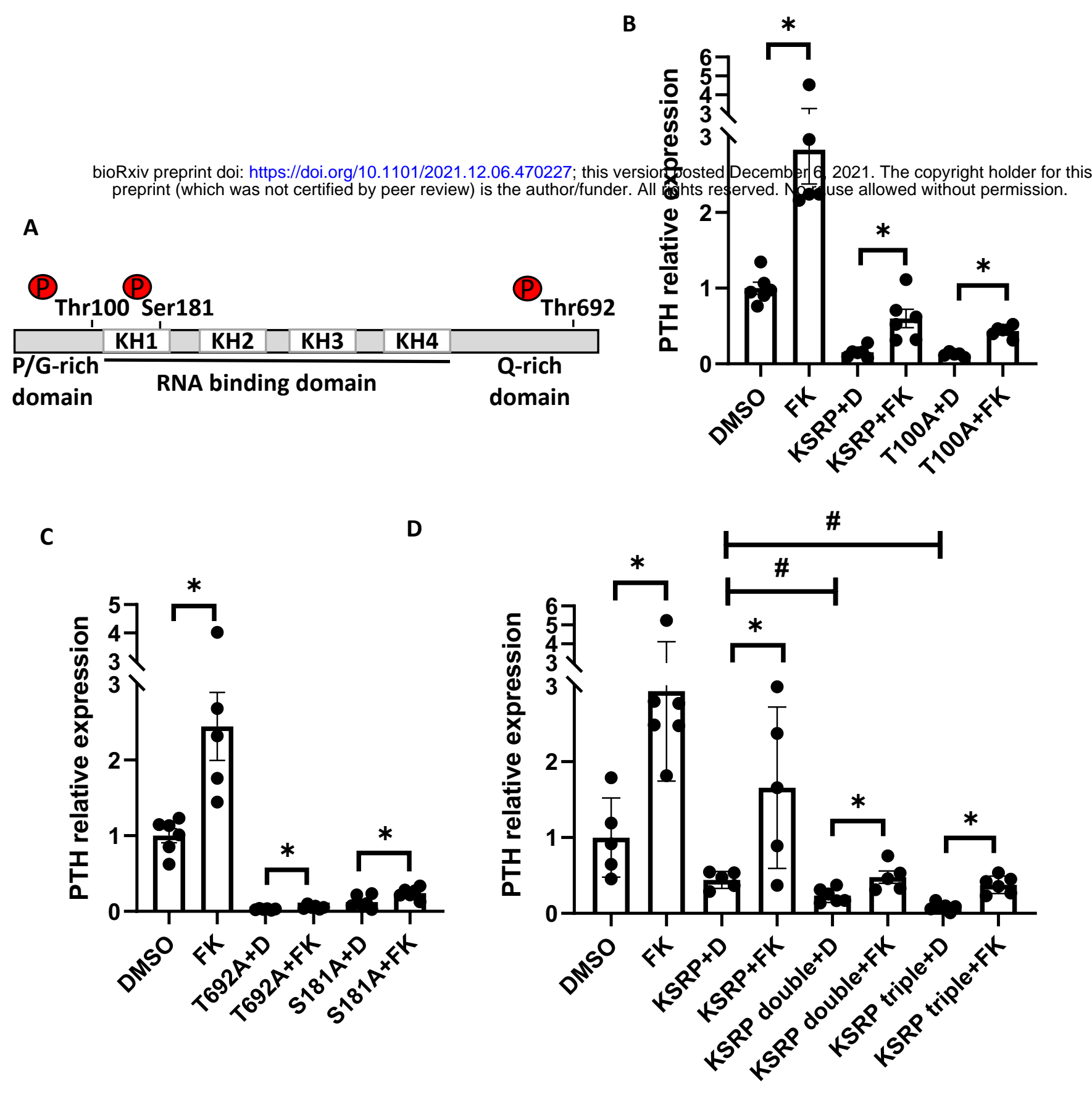

Figure 8. Pin1 induced changes in KSRP phosphorylation alter PTH expression. A. Schematic representation of the primary structure of KSRP, including the four hnRNP K homology (KH) domains and position of the relevant phosphorylation sites. (B-D) HEK293 cells were transiently transfected with expression plasmids for human PTH without and with native KSRP or KSRP phosphorylation null single mutants Thr100Ala (T100A) (A); Thr692Ala (T692A) or Ser181 Ala S181A) (B); KSRP Ser181Ala;Thr100Ala (double) or Ser181Ala;Thr100Ala;Thr692Ala (triple) mutants (C). Transfected cells were incubated with forskolin (FK) or vehicle DMSO (+D). PTH mRNA levels at $24 \mathrm{~h}$, normalized to GAPDH, were determined by qRT-PCR. Results are presented as mean $\pm \mathrm{SE}$ of fold change, compared cells transfected with PTH expression plasmid and a control empty vector. *, p $<0.05$ forskolin vs DMSO; \#, vs KSRP+D. 
A

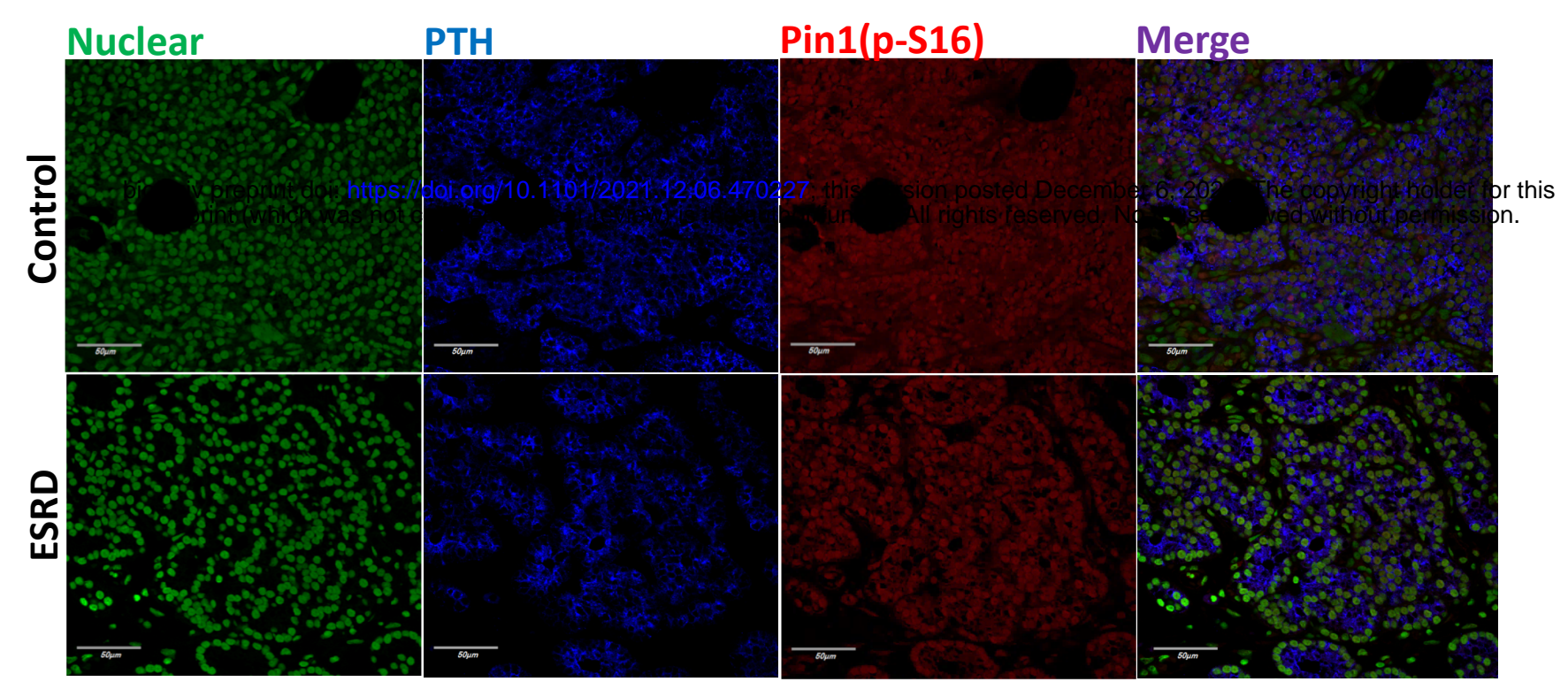

B

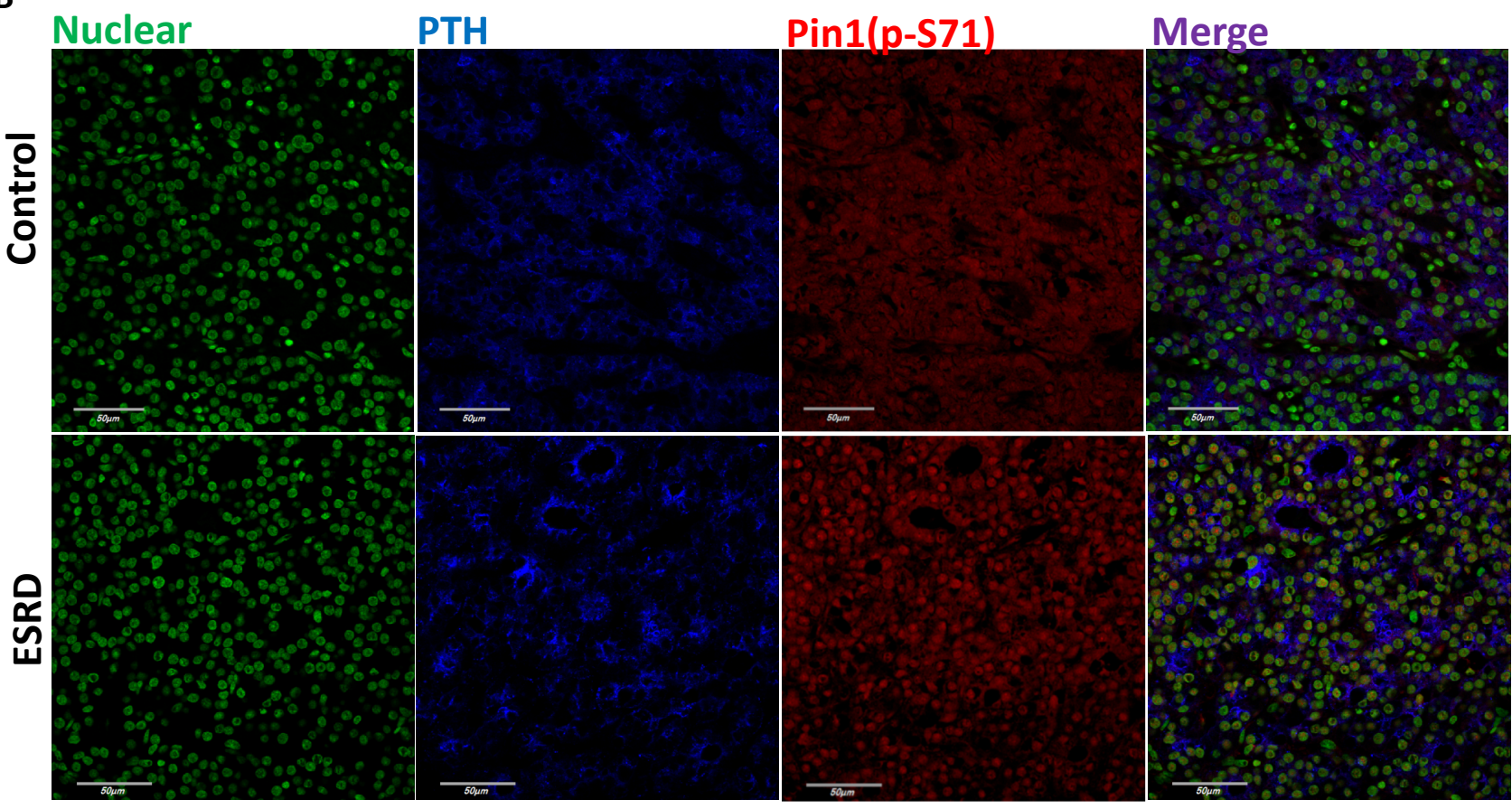

Fig 9. Pin1 phosphorylation in human parathyroid sections from control and end stage renal disease patients. Representative parathyroid sections from end stage renal disease (ESRD) patients and control parathyroids removed at total thyroidectomy were stained by immunofluorescence for sytox nuclear staining (green) and antibodies for PTH (blue), Pin1 pSer16 (A) or p-Ser71 (B) (red) and merge (purple). Similar results were obtained in additional 3 control and CKD patients for pSer16 and 2 control and 3 CKD patients for pSer71. 


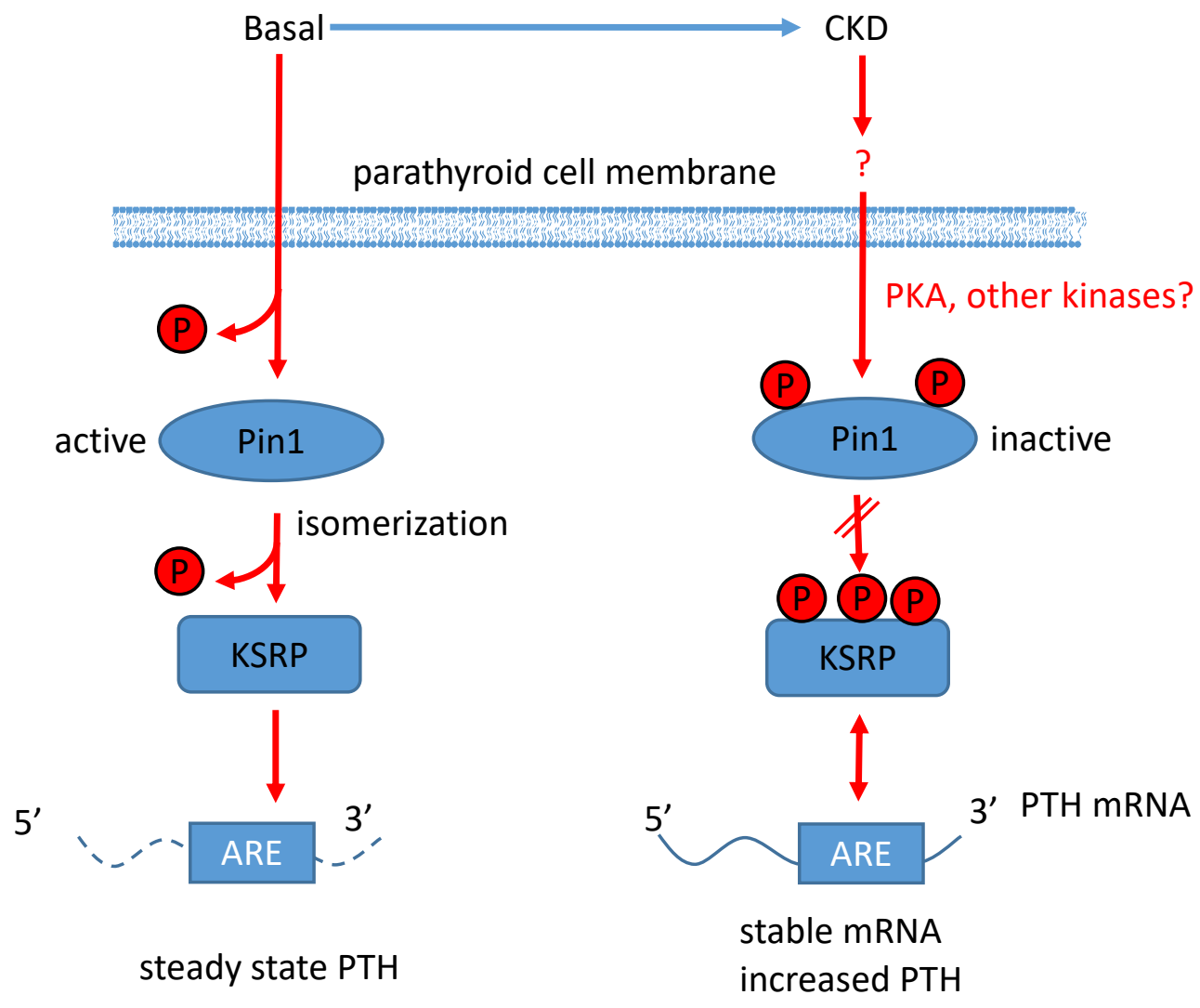

Figure 10. Model for the changes induced by CKD in the parathyroid cells that lead to increased PTH gene expression in SHP. Under basal conditions, Pin1 is unphosphorylated at Ser16 and 71 and thus active, leading to conformational change induced decrease in KSRP phosphorylation. Unphosphorylated KSRP binds to the adenine uridine rich element (ARE) in the PTH mRNA 3'-untranslated region and along with other PTH mRNA binding proteins determines steady state PTH levels. CKD induces Pin1 phosphorylation and hence decreased isomerase activity, leaving KSRP phosphorylated at three Pin1 target sites. Phosphorylated KSRP fails to bind PTH mRNA and induce its degradation, resulting in increased PTH mRNA and serum levels. 